\title{
Stable two-plane focusing for emittance-dominated sheet-beam transport
}

\author{
B. E. Carlsten, L. M. Earley, F. L. Krawczyk, and S. J. Russell \\ Los Alamos National Laboratory, Los Alamos, New Mexico, USA \\ J. M. Potter \\ JP Accelerator Works, Los Alamo, New Mexico, USA \\ P. Ferguson \\ MDS Company, Oakland, California, USA
}

S. Humphries, Jr.

Field Precision, Albuquerque, New Mexico, USA

(Received 26 April 2005; published 29 June 2005)

\begin{abstract}
Two-plane focusing of sheet electron beams will be an essential technology for an emerging class of high-power, 100 to $300 \mathrm{GHz}$ rf sources [Carlsten et al., IEEE Trans. Plasma Sci. 33, 85 (2005)]. In these devices, the beam has a unique asymmetry in which the transport is emittance dominated in the sheet's thin dimension and space-charge dominated in the sheet's wide dimension. Previous work has studied the stability of the transport of beams in the emittance-dominated regime for both wiggler and periodic permanent magnet (PPM) configurations with single-plane focusing, and has found that bigger envelope scalloping occurs for equilibrium transport, as compared to space-charge dominated beams [Carlsten et al., this issue, Phys. Rev. ST Accel. Beams 8, 062001 (2005)]. In this paper, we describe the differences in transport stability when two-plane focusing is included. Two-plane wiggler focusing degrades the transport stability slightly, whereas two-plane PPM focusing greatly compromises the transport. On the other hand, single-plane PPM focusing can be augmented with external quadrupole fields to provide weak focusing in the sheet's wide dimension, which has stability comparable to two-plane wiggler transport.
\end{abstract}

DOI: 10.1103/PhysRevSTAB.8.062002

PACS numbers: $41.75 . \mathrm{Ht}$

\section{INTRODUCTION}

A new need for high-frequency, high-power rf sources has been emerging for advanced radar and communications, with frequencies in the band between 100 and $300 \mathrm{GHz}$ and peak powers as high as several hundreds of kilowatts, and with bandwidths of up to $10 \%$. Previous work has indicated that a sheet-beam driven travelingwave tube (TWT) can meet this need [1-3]. Because of the extremely small dimensions required $(0.5 \mathrm{~mm}$ or less in the sheet's thin dimension), stability of sheet-beam transport has been recognized as one of the key technologies required for this type of TWT, in addition to rf mode control in these highly overmoded structures.

We have analytically and numerically studied the transport of a sheet beam, mostly for the nominal parameters of a 20-A, 120-kV beam with elliptical cross section $1 \mathrm{~cm}$ by $0.5 \mathrm{~mm}$. This type of beam is unique because the dynamics are space-charge dominated in the wide dimension but emittance dominated in the thin emittance, requiring strong focusing in that dimension. Some focusing is still required in the wide dimension, because the relatively weak spacecharge force is still strong enough to expand the beam to an unacceptable level. A previous analysis of single-plane focusing in the emittance-dominated regime [4] reproduced earlier results of stability based on the Mathieu equation for space-charge dominated beams $[5,6]$, and additionally showed that an emittance-dominated beam has much greater envelope scalloping than a space-charge dominated beam which can lead to unacceptable interception in narrow beam tunnels.

In this paper, we extend the analysis of an emittancedominated beam to include two-plane focusing, of both wiggler and permanent magnet (PPM) focusing configurations. We find that two-plane focusing with a wiggler configuration maintains most of the stability of singleplane wiggler focusing, but two-plane PPM focusing requires use of an external quadrupole field to maintain good beam stability. We first review the key results of the earlier analysis [4] in this Introduction before we begin the twoplane analysis.

In general, the magnetic field for a planar focusing array can be expressed as [7]

$$
B=-\nabla \chi_{m}
$$

where

$$
\chi_{m}=\frac{B_{w}}{k_{z}} \cosh \left(k_{x} x\right) \cos \left(k_{z} z\right)\left[a \sinh \left(k_{y} y\right)+b \cosh \left(k_{y} y\right)\right]
$$

where $k_{z}$ is $2 \pi$ divided by the focusing period $L$. The $\cosh \left(k_{x} x\right)$ term is typically introduced by shaping of the magnets or pole pieces. The wave equation relates the wave 
numbers, and gives $k_{x}^{2}+k_{y}^{2}=k_{z}^{2}$. Here we assume the beam motion is in $\hat{z}$, the beam is narrow in $\hat{y}$ and wide in $\hat{x} . B_{w}$ is used to denote the peak field, to be consistent with previous work from the free-electron laser community. In Eq. (2), we use the parameters $a$ and $b$ to differentiate between a wiggler field $(a=1, b=0)$ and a PPM field $(a=0, b=1)$. (Only the PPM field exists with cylindrical symmetry.) This potential gives us these field components:

$$
\begin{aligned}
& B_{x}=-\frac{k_{x}}{k_{z}} B_{w} \sinh \left(k_{x} x\right) \cos \left(k_{z} z\right)\left[a \sinh \left(k_{y} y\right)+b \cosh \left(k_{y} y\right)\right], \\
& B_{y}=-\frac{k_{y}}{k_{z}} B_{w} \cosh \left(k_{x} x\right) \cos \left(k_{z} z\right)\left[a \cosh \left(k_{y} y\right)+b \sinh \left(k_{y} y\right)\right], \\
& B_{z}=B_{w} \cosh \left(k_{x} x\right) \sin \left(k_{z} z\right)\left[a \sinh \left(k_{y} y\right)+b \cosh \left(k_{y} y\right)\right] .
\end{aligned}
$$

When $k_{x}=0, B_{y}$ and $B_{z}$ are independent of horizontal position, and $B_{x}$ vanishes. This leads to only focusing in the $\hat{y}$ plane, which in the free-electron laser community has been known as "natural focusing." With $k_{x} \neq 0$, there is horizontal focusing of the beam in addition to vertical focusing, which is known as two-plane focusing. For real transport sections, some horizontal focusing will always be needed due to the space-charge fields of the beam. For the type of sheet beam we are concerned with, the horizontal focusing required is well over an order of magnitude smaller than the vertical focusing. Because the required horizontal force for this example (and many other practical cases) is so small, there are several options in how it can be produced, some of which we will describe in later sections of this paper.

The single-particle transverse equation of motion with natural focusing becomes

$$
\begin{aligned}
\ddot{y}= & -\left(\frac{e}{m \gamma}\right)^{2} \frac{B_{w}^{2}}{k_{w}}\left[a \cosh \left(k_{w} y\right)+b \sinh \left(k_{w} y\right)\right] \\
& \times\left[a \sinh \left(k_{w} y\right)+b \cosh \left(k_{w} y\right)\right] \cos ^{2}\left(k_{w} z\right) \\
& + \text { space charge force, }
\end{aligned}
$$

where we use $k_{w}=k_{z}=k_{y}$, and where the first term in brackets is from the horizontal motion of the beam $v_{x}$, and the second term is from $B_{z}$. For small $y, \cosh \left(k_{w} y\right)=1$ and $\sinh \left(k_{w} y\right)=k_{w} y$, and if $a=1, b=0$ or $a=0, b=1$, we end up with linear focusing in $y$ and recover the same equation of motion for both PPM and wiggler focusing. For emittance-dominated transport, the space-charge force can be neglected altogether, and we recover the Mathieu equation for the cases where either $a=1, b=0$ or $a=0$, $b=1$ :

$$
\ddot{y}+y\left(\frac{e}{m \gamma}\right)^{2} B_{w}^{2} \cos ^{2}\left(k_{w} z\right)(a+b)=0 .
$$

The stability of a Mathieu equation is well known. The trajectory described by it is stable as long as the Mathieu parameter $\alpha$, defined for natural focusing by

$$
\alpha=\frac{B_{w}^{2}}{2}\left(\frac{e}{m c \gamma \beta}\right)^{2}\left(\frac{L}{2 \pi}\right)^{2},
$$

is less than 0.66. Using this equation of motion, we can derive the envelope equations [4], which, for natural focusing, become

$$
\begin{gathered}
X^{\prime \prime}=\frac{I / I_{A}}{\gamma^{3} \beta^{3}} \frac{1}{X+Y}+\frac{\varepsilon_{x, \text { norm }}^{2}}{X^{3} \gamma^{2} \beta^{2}}, \\
Y^{\prime \prime}=\frac{I / I_{A}}{\gamma^{3} \beta^{3}} \frac{1}{X+Y}+\frac{\varepsilon_{y, \text { norm }}^{2}}{Y^{3} \gamma^{2} \beta^{2}}-Y\left(\frac{e}{\beta c m \gamma}\right)^{2} \frac{B_{w}^{2}}{2} .
\end{gathered}
$$

We can identify the first term on the right hand side as a space-charge term, the second term as an emittance term, and, for the vertical motion, the third term as the focusing term from the planar magnetic structure. At $120 \mathrm{kV}, \gamma$ is 1.235 and $\gamma \beta$ is 0.724 , and for the nominal Los Alamos design [1], the normalized beam emittance is $1.35 \mu \mathrm{m}$ and the current is $20 \mathrm{~A}$. For a 10 by $0.5 \mathrm{~mm}$ beam, $Y$ is $0.125 \mathrm{~mm}$ and $X$ is $2.5 \mathrm{~mm}$, the focusing term is $40.9 \mathrm{~m}^{-1}$ times $B_{w}^{2}$ in Tesla, the space-charge term is $1.18 \mathrm{~m}^{-1}$ (both horizontal and vertical envelope equation), and the emittance term is $1.79 \mathrm{~m}^{-1}$ (and $8.9 \times 10^{-4} \mathrm{~m}^{-1}$ for the horizontal envelope equation). Even for this low emittance, the emittance term dominates in the vertical dimension (and is negligible in the horizontal dimension). This transverse asymmetry is unique for sheet beams, and is more pronounced for larger emittance cases.

The required focusing strength in the vertical dimension for these parameters is $0.266 \mathrm{~T}$. With just the emittance (ignoring the space-charge force), the magnetic field strength required for equilibrium flow would need to be $0.206 \mathrm{~T}$, and with just the space-charge force, the magnetic field strength would need to be $0.168 \mathrm{~T}$. Equating the focusing part of the envelope equation to the emittance part, we find that the required peak field for an emittancedominated beam is given by

$$
B_{\text {req }}=\sqrt{2} \varepsilon_{y, \text { norm }} \frac{m c}{Y^{2} e} .
$$

Note that the required field does not depend on the beam energy at all. Relating the field required for force balance to the Mathieu parameter $\alpha$ we find

$$
\alpha=\frac{\varepsilon_{y, \text { norm }}^{2}}{\gamma^{2} \beta^{2}} \frac{1}{Y^{4}}\left(\frac{L}{2 \pi}\right)^{2} .
$$

Requiring that $\alpha$ must be less than 0.66 puts a relation between the beam emittance, focusing period, and beam width. The maximum allowable focusing period given the beam emittance and size is then

$$
L_{\max }=2 \pi \sqrt{0.66} \frac{\gamma \beta}{\varepsilon_{y, \text { norm }}} Y^{2} .
$$

Because of physical limitations in permanent magnets, the period typically cannot be much smaller than 5 to 
$10 \mathrm{~mm}$. In addition, the maximum allowable rms beam size $Y$ is proportional to the $\mathrm{rf}$ wavelength for $\mathrm{rf}$ generation sources. With these constraints, Eq. (11) emphasizes the need for having a low-emittance beam to be able to transport a beam in a high-frequency rf source.

In contrast to a space-charge dominated beam, the beam envelope of an emittance-dominated beam undergoes large amplitude scalloping in the vertical dimension well before the stability limit is reached [4]. In Fig. 1 we plot the amplitude of the envelope ripple [peak to peak, normalized to the equilibrium radius from Eq. (8)] as a function of the Mathieu parameter, for both wiggler and PPM focusing, for both the cases of an emittance-dominated beam and a space-charge dominated beam.

Surprisingly, the addition of space charge stabilizes the flow, resulting in much lower envelope scalloping. The increased ripple amplitude for wiggler focusing at the same focusing strength (or $\alpha$ ) makes it much less desirable for transport than does PPM focusing. Note the beam transport in the wiggler field becomes unstable for $\alpha$ slightly larger than 0.3 .

Equations (9) and (10) and Figs. 1 also point out a significant difference between the transport of a spacecharge dominated beam and an emittance-dominated beam in an rf tube. Both as the beam is bunched, causing local increases in the beam current, and as rf power is extracted from a space-charge dominated beam, the space-charge forces increase and a greater magnetic field is required to maintain the same equilibrium beam radius. As a result, it is common that the magnetic field of a PPM or wiggler structure is designed to be greater than that required for an unmodulated beam. However, from Eq. (9) we see that the required confining magnetic field for an emittance-dominated beam is unchanged both as the beam is bunched and as power is extracted from it (as long as the transverse forces are small enough that the unnormalized emittance does not change). Additionally from

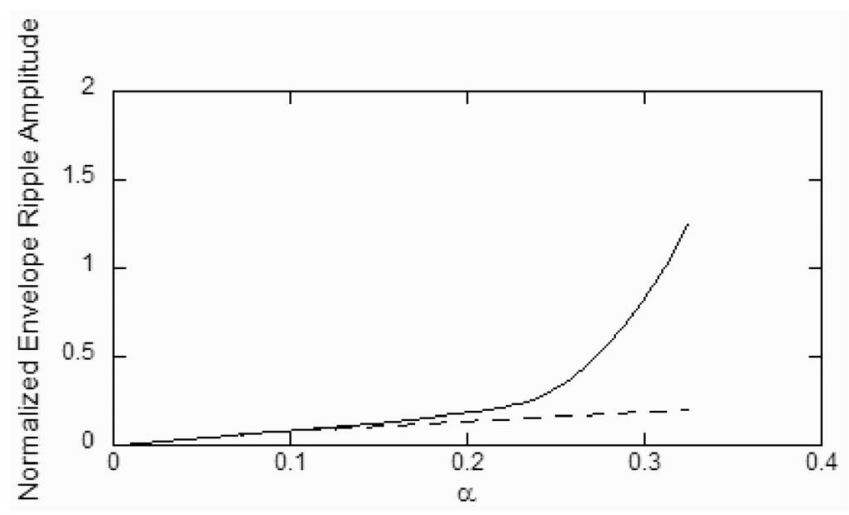

(a)
Eq. (10), we see that as the beam loses energy, the Mathieu parameter $\alpha$ increases, which leads to larger amplitude beam envelope rippling.

Although the $a b$ cross terms in Eq. (4) introduce nonlinear terms, numerical calculations of transport with nonzero cross terms show no emittance growth, as shown in Fig. 2 for a $2 / 3$ wiggler, $1 / 3$ PPM field split. In these and the following figures, 4 times the rms beam size is plotted, for both the vertical and horizontal dimensions, because this equals the full beam size for a hard-edged uniformdensity beam in both the space-charge and emittancedominated regimes, and is a good measure of effective beam size for a nonuniform-density beam.

Although natural focusing provides good linearity and virtually no emittance growth, it does not provide adequate horizontal focusing. For the example of the Los Alamos sheet beam, with only natural focusing and a peak magnetic field of $0.266 \mathrm{~T}$, the beam expands significantly in the horizontal direction because of the horizontal space-charge force, also affecting the focusing in the vertical direction, Fig. 3. To control this expansion, two-plane focusing must be included in the focusing structure.

The goal of this paper is to understand the changes in stability from natural focusing as shown in Fig. 1, when two-plane focusing is included. This is described in detail in the following four sections. In the next section, Sec. II, we develop a two time scale description of particle orbits using two-plane focusing as described by Eqs. (1) and (2), following $[7,8]$. This development is used to develop transverse equation of motions for both two-plane PPM and wiggler focusing and yields expression we can use to find the needed ratio of $k_{x} / k_{y}$ for balanced two-plane focusing. The longitudinal velocity modulation is then derived for both two-plane PPM and wiggler focusing in Sec. III, which shows that longitudinal velocity is constant (averaged over a wiggle period), for both field profiles, impor-

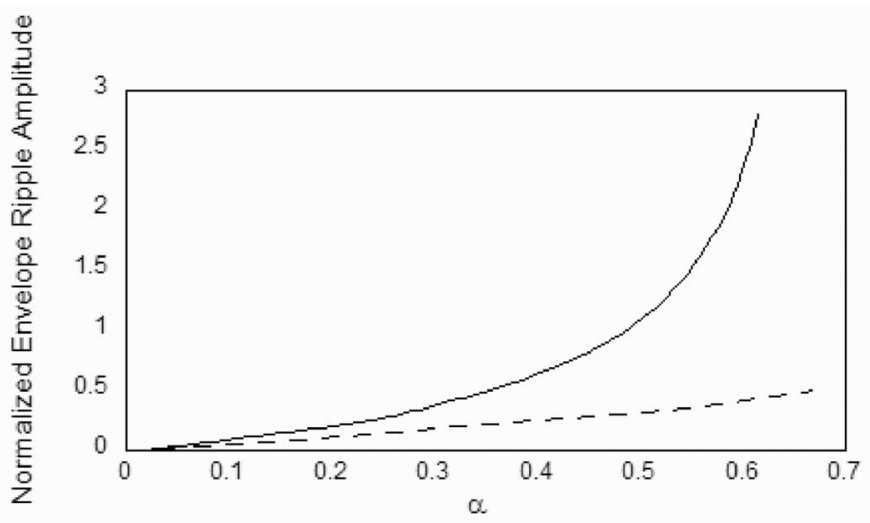

(b)

FIG. 1. Envelope ripple amplitude versus focusing period, solid line for emittance-dominated beam, dashed line for space-charge dominated beam. (a) Wiggler focusing. (b) PPM focusing. 


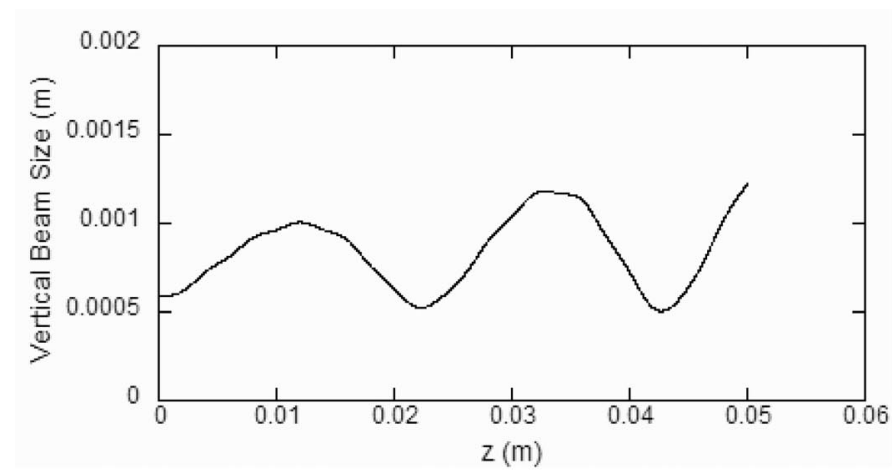

(a)

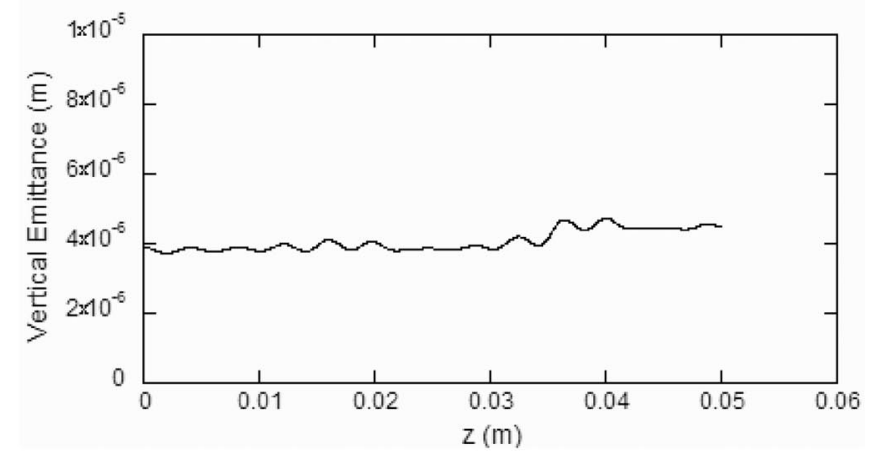

(b)

FIG. 2. (a) Vertical beam size versus axial distance for a $2 / 3$ wiggler, $1 / 3$ PPM field split. (b) Vertical emittance versus axial distance.

tant to maintain phase coherency of $\mathrm{rf}$ generation. In Sec. IV, two-plane focusing transport is numerically investigated for both PPM and wiggler focusing, for a lowemittance case. We show that the wiggler field stability is mostly unchanged with two-plane focusing, but we find that the beam transport in a PPM field is greatly degraded with two-plane focusing, especially if the beam has a very large aspect ratio. It is determined that the poor transport is due to the nonlinearities in the force that particles experience towards the horizontal edges of the beam, at least partly arising from the $\sinh \left(k_{x} x\right)$ term in $B_{x}$. In Sec. V, we compare two-plane wiggler focusing with two alternative two-plane PPM focusing schemes, where the PPM structure has only natural focusing and the horizontal focusing is added either by a uniform quadrupole structure or by a periodic quadrupole structure. These schemes are compared to two-plane wiggler focusing, for a higher emittance case. Good transport stability is found with the uniform quadrupole field, but the transport is less stable with a periodic external quadrupole field. This is compared to earlier work studying PPM focusing augmented by uni- form and by periodic quadrupole fields [6] for zeroemittance beams and short focusing periods.

\section{WIGGLE-AVERAGED EQUATIONS OF MOTION}

The purpose of this section is to derive expressions we can use to find the ratio $k_{x} / k_{y}$ for balanced two-plane focusing, as a function of beam emittance and current. These expressions will be used in the following sections for designing two-plane focusing schemes.

We can improve the "natural focusing" by providing focusing in both transverse dimensions by curving the pole pieces [8], offsetting the wiggler magnets [9], notching the wiggler magnets $[10,11]$, or external quadrupole focusing [9]. Two-plane focusing was developed for emittancedominated round electron beams intended for free-electron lasers (FELs), where the beam is transported and focused in a wiggler field. For sheet beams, there is much less horizontal defocusing, but even for the nominal case used here, two-plane focusing is needed, as seen in Fig. 3.

To understand the particle equation of motion with twoplane focusing, we need to wiggle average the motion.
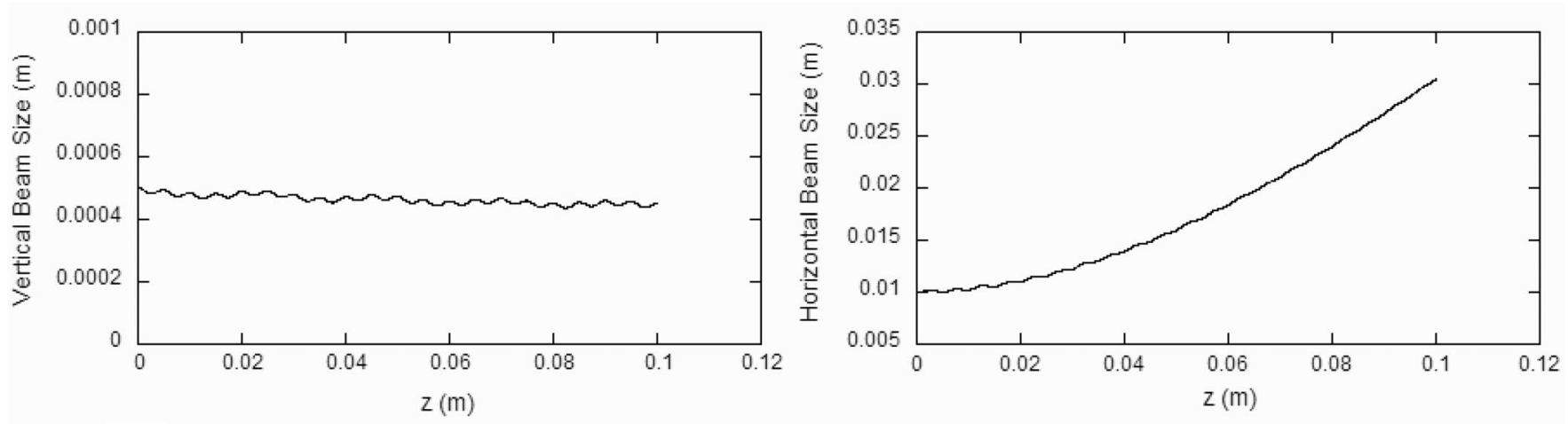

(b)

FIG. 3. (a) Vertical beam size, for the nominal natural focusing case (emittance of $1.4 \mathrm{~mm}, 20 \mathrm{~A}, 0.266 \mathrm{~T}$ wiggler focusing field). (b) Horizontal beam size. 
Scharlemann [8] did this first for wiggler fields, and Booske [7] extended this for PPM focusing. We will do this slightly differently by following both components of the field (wiggler and PPM) simultaneously, so we can compare them directly. Wiggle averaging removes the Mathieu equation in the particle equation of motion - we need to remember the Mathieu stability regions still exist, although the stability regions will shift. The goal of this section is to derive the vertical and horizontal equations of motion for both two-plane PPM and wiggler focusing, which, in turn, can be used to design two-plane focusing configurations for specific beam parameters. One important result is that the wiggler configuration has much greater horizontal focusing, for similar $B_{x}$ field strengths. This, in turn, has consequences on emittance growth from nonlinear field components, which we will see in Sec. IV.

We will consider the lowest Fourier component of the field as described in Eqs. (1)-(3). Since we are most concerned with focusing the beam vertically, where the beam is emittance dominated, we will use particle equa- tions of motion without space charge,

$$
\begin{aligned}
\ddot{x} & =\frac{e}{m \gamma}\left(\dot{y} B_{z}-\dot{z} B_{y}\right), \quad \ddot{y}=\frac{e}{m \gamma}\left(\dot{z} B_{x}-\dot{x} B_{z}\right), \\
\ddot{z} & =\frac{e}{m \gamma}\left(\dot{x} B_{y}-\dot{y} B_{x}\right) .
\end{aligned}
$$

The dynamics are too complicated to solve exactly, so following $[7,8]$, we use a two-time scale solution:

$$
x=x_{0}+x_{1}, \quad y=y_{0}+y_{1},
$$

where the $x_{0}$ motion is gradual (variations on the axial scale of the natural focusing) and the $x_{1}$ motion goes as $\cos \left(k_{z} z\right)$. Our approach will be to (i) find $x_{1}(z)$ and $y_{1}(z)$ assuming $x_{0}$ and $y_{0}$ are constant, then (ii) using the $x_{1}(z)$ and $y_{1}(z)$ we found, use the full equation of motion to find $x_{0}(z)$ and $y_{0}(z)$, using wiggle-period averaging to simplify the calculation, and (iii) finally add in the space-charge force.

The transverse equations of motion are

$$
\begin{aligned}
\ddot{x}_{0}+\ddot{x}_{1}= & \frac{e}{m \gamma}\left\{\left(\dot{y}_{0}+\dot{y}_{1}\right) B_{w} \cosh \left(k_{x} x\right) \sin \left(k_{z} z\right)\left[a \sinh \left(k_{y} y\right)+b \cosh \left(k_{y} y\right)\right]+\left(\dot{z}_{0}+\dot{z}_{1}\right) B_{w} \frac{k_{y}}{k_{z}} \cosh \left(k_{x} x\right) \cos \left(k_{z} z\right)\right. \\
& \left.\times\left[a \cosh \left(k_{y} y\right)+b \sinh \left(k_{y} y\right)\right]\right\}, \\
\ddot{y}_{0}+\ddot{y}_{1}= & \frac{e}{m \gamma}\left\{-\left(\dot{z}_{0}+\dot{z}_{1}\right) B_{w} \frac{k_{x}}{k_{z}} \sinh \left(k_{x} x\right) \cos \left(k_{z} z\right)\left[a \sinh \left(k_{y} y\right)+b \cosh \left(k_{y} y\right)\right]+\left(\dot{x}_{0}+\dot{x}_{1}\right) B_{w} \cosh \left(k_{x} x\right) \sin \left(k_{z} z\right)\right. \\
& \left.\times\left[a \sinh \left(k_{y} y\right)+b \cosh \left(k_{y} y\right)\right]\right\}
\end{aligned}
$$

We will use $\dot{z}_{0} \gg \dot{z}_{1}, \dot{x}_{0}$ and $\dot{x}_{1}, x_{0} \gg x_{1}$, and $d / d t=\dot{z}_{0}(d / d z)$.

The fast time scales are dominated by the $\dot{z}_{0}$ terms:

$$
\begin{aligned}
& \ddot{x}_{1}=\frac{e \dot{z}_{0}}{m \gamma} B_{w} \frac{k_{y}}{k_{z}} \cosh \left(k_{x} x\right) \cos \left(k_{z} z\right)\left[a \cosh \left(k_{y} y\right)+b \sinh \left(k_{y} y\right)\right], \\
& \dot{x}_{1}=\frac{e}{m \gamma} B_{w} \frac{k_{y}}{k_{z}^{2}} \cosh \left(k_{x} x\right) \sin \left(k_{z} z\right)\left[a \cosh \left(k_{y} y\right)+b \sinh \left(k_{y} y\right)\right], \\
& x_{1}=-\frac{e}{m \gamma \dot{z}_{0}} B_{w} \frac{k_{y}}{k_{z}^{3}} \cosh \left(k_{x} x\right) \cos \left(k_{z} z\right)\left[a \cosh \left(k_{y} y\right)+b \sinh \left(k_{y} y\right)\right], \\
& \ddot{y}_{1}=-\frac{e \dot{z}_{0}}{m \gamma} B_{w} \frac{k_{x}}{k_{z}} \sinh \left(k_{x} x\right) \cos \left(k_{z} z\right)\left[a \sinh \left(k_{y} y\right)+b \cosh \left(k_{y} y\right)\right], \\
& \dot{y}_{1}=-\frac{e}{m \gamma} B_{w} \frac{k_{x}}{k_{z}^{2}} \sinh \left(k_{x} x\right) \sin \left(k_{z} z\right)\left[a \sinh \left(k_{y} y\right)+b \cosh \left(k_{y} y\right)\right] \\
& y_{1}=\frac{e}{m \gamma \dot{z}_{0}} B_{w} \frac{k_{x}}{k_{z}^{3}} \sinh \left(k_{x} x\right) \cos \left(k_{z} z\right)\left[a \sinh \left(k_{y} y\right)+b \cosh \left(k_{y} y\right)\right] .
\end{aligned}
$$

With small argument expansions, $y_{1}=\left(e / m \gamma \dot{z}_{0}\right) B_{w}\left(k_{x}^{2} / k_{z}^{3}\right) x \cos \left(k_{z} z\right)\left(a k_{y} y+b\right)$. For a high-aspect-ratio sheet beam, we expect $k_{y}$ is significantly larger than $k_{x}$, however, depending on the actual horizontal width of the beam $k_{x} x$ may be much larger than $k_{y} y$. The $\dot{x}_{1}$ term is on the order of unity for a wiggler field and on the order of $k_{y} y$ for a PPM field, whereas $\dot{y}_{1}$ is on the order of $k_{y} y k_{x} x$ for a wiggler field and on the order of $k_{x} x$ for a PPM field. This means that $\dot{x}_{1} \gg \dot{y}_{1}$ in a wiggler field, but $\dot{x}_{1} \sim \dot{y}_{1}$ in a PPM field. For the $\ddot{x}_{0}, \ddot{y}_{0}$ terms, we use the full equations of motion: 


$$
\begin{aligned}
\ddot{x}_{0}= & \frac{e}{m \gamma}\left(-\frac{e}{m \gamma} B_{w}^{2} \frac{k_{x}}{k_{z}^{2}} \sin ^{2}\left(k_{z} z\right)\left\{\left[k_{x}\left(x_{0}+x_{1}\right)\right]\left(1+k_{x}^{2} x_{0} x_{1}\right)\right\}\right. \\
& \times\left[a \sinh \left(k_{y} y\right)+b \cosh \left(k_{y} y\right)\right]^{2}+\dot{z}_{0} B_{w} \frac{k_{y}}{k_{z}}\left(1+k_{x}^{2} x_{0} x_{1}\right) \\
& \left.\times \cos \left(k_{z} z\right)\left[a\left(1+k_{y}^{2} y_{0} y_{1}\right)+b k_{y}\left(y_{0}+y_{1}\right)\right]\right)
\end{aligned}
$$

where we used $\cosh \left(k_{x} x\right) \approx 1+\left(k_{x} x\right)^{2} / 2=1+k_{x}^{2}\left(x_{0}+\right.$ $\left.x_{1}\right)^{2} / 2 \approx 1+k_{x}^{2} x_{0} x_{1}$ because we can drop the $k_{x}^{2} x_{0}^{2} / 2$ part when compared to the unity term. Also, $\sinh \left(k_{x} x\right)=k_{x} x$.

Since $\sin ^{2}\left(k_{z} z\right)$ averages to $\frac{1}{2}$, the first term in Eq. (16) becomes $-(e / m \gamma)^{2} B_{w}^{2}\left(k_{x}^{2} / k_{z}^{2}\right)\left(x_{0} b^{2} / 2\right)$ (the $a$ term is higher order, and we have dropped $a b$ cross terms)

The second term in Eq. (16) is rewritten as

$$
\begin{aligned}
& \frac{e}{m \gamma} B_{w} \frac{k_{y}}{k_{z}} \cos \left(k_{z} z\right)\left[a\left(1+k_{x}^{2} x_{0} x_{1}+k_{y}^{2} y_{0} y_{1}\right)\right. \\
& \left.+b\left(k_{y} y_{0} k_{x}^{2} x_{0} x_{1}+k_{y} y_{1}\right)\right] \\
& =\frac{e}{m \gamma} B_{w} \frac{k_{y}}{k_{z}} \frac{1}{2}\left[a k_{x}^{2} x_{0}\left(-\frac{e}{m \gamma \dot{z}_{0}} B_{w} \frac{k_{y}}{k_{z}^{3}} a\right)\right. \\
& \left.\quad+b y_{0}\left(\frac{e}{m \gamma \dot{z}_{0}} B_{w} \frac{k_{x}}{k_{z}^{3}} k_{x} x_{0} b\right)\right] \\
& =\left(\frac{e}{m \gamma}\right)^{2} B_{w}^{2} \frac{k_{y}}{k_{z}} \frac{1}{2}\left(-a^{2}+b^{2}\right) k_{x}^{2} x_{0} \frac{k_{y}}{k_{z}^{3}},
\end{aligned}
$$

where we have used the fact that $y_{1} \ll x_{1}$ if $a=1$ and we have again dropped the small terms. Combining these terms, we have

$$
\ddot{x}_{0}=-\left(\frac{e}{m \gamma}\right)^{2} B_{w}^{2} \frac{1}{2} \frac{k_{x}^{2}}{k_{z}^{2}}\left(a^{2} \frac{k_{y}^{2}}{k_{z}^{2}}+b^{2} \frac{k_{x}^{2}}{k_{z}^{2}}\right) x_{0} .
$$

So the horizontal focusing for the wiggler and PPM cases are similar, but since $k_{x}$ is smaller than $k_{y}$ for cases of interest to us, the PPM field will tend to have much less horizontal focusing. Recall that we need to add in the space-charge defocusing (if space charge is not negligible), and we need to recall the Mathieu stability (which is hidden because of the averaging).

For the $\ddot{y}_{0}$ equation, we get pretty much the same thing, starting with

$$
\begin{aligned}
\ddot{y}_{0}= & \frac{e}{m \gamma}\left\{-\dot{z}_{0} B_{w} \frac{k_{x}}{k_{z}} \sinh \left(k_{x} x\right) \cos \left(k_{z} z\right)\left[a \sinh \left(k_{y} y\right)\right.\right. \\
& \left.+b \cosh \left(k_{y} y\right)\right]-\dot{x}_{1} B_{w} \cosh \left(k_{x} x\right) \sin \left(k_{z} z\right) \\
& \left.\times\left[a \sinh \left(k_{y} y\right)+b \cosh \left(k_{y} y\right)\right]\right\}
\end{aligned}
$$

or

$$
\begin{aligned}
\ddot{y}_{0}= & \frac{e}{m \gamma}\left\{-\dot{z}_{0} B_{w} \frac{k_{x}}{k_{z}}\left[k_{x}\left(x_{0}+x_{1}\right)\right] \cos \left(k_{z} z\right)\left[a k_{y}\left(y_{0}+y_{1}\right)\right.\right. \\
& \left.+b\left(1+k_{y}^{2} y_{0} y_{1}\right)\right]-\frac{e}{m \gamma} B_{w}^{2} \frac{k_{y}}{k_{z}^{2}}(1 \\
& \left.\left.+k_{x}^{2} x_{0} x_{1}\right)^{2} \sin ^{2}\left(k_{z} z\right)\left(a+b k_{y} y_{0}\right)\left(a k_{y} y+b_{0}\right)\right\} .
\end{aligned}
$$

$$
\begin{aligned}
- & \frac{e}{m \gamma} \dot{z}_{0} B_{w} \frac{k_{x}}{k_{z}}\left[k_{x} x_{1}\left(a k_{y} y_{0}+b\right)+k_{x} x_{0}\left(a k_{y} y_{1}+b k_{y}^{2} y_{0} y_{1}\right)\right] \cos \left(k_{z} z\right) \\
& =-\frac{e}{m \gamma} \dot{z}_{0} B_{w} \frac{k_{x}}{k_{z}}\left\{a\left(k_{x} x_{1} k_{y} y_{0}+k_{x} x_{0} k_{y} y_{1}\right)+b\left(k_{x} x_{1}+k_{x} x_{0} k_{y}^{2} y_{0} y_{1}\right)\right\} \\
& =-\frac{e}{m \gamma} \dot{z}_{0} B_{w} \frac{k_{x}}{k_{z}}\left\{a k_{x} k_{y} y_{0}\left(-\frac{e}{m \gamma}\right) \frac{B_{w}}{\dot{z}_{0}} \cos \left(k_{z} z\right) a \frac{k_{y}}{k_{z}^{3}}+b k_{x}\left(-\frac{e}{m \gamma}\right) \frac{k_{y}}{k_{z}^{3}} \frac{B_{w}}{\dot{z}_{0}} \cos \left(k_{z} z\right) k_{y} y_{0} b\right\} \\
& =-\left(\frac{e}{m \gamma}\right)^{2} \frac{B_{w}^{2}}{2} \frac{k_{x}}{k_{z}}\left(-a^{2} \frac{k_{x} k_{y}^{2}}{k_{z}^{3}}-b^{2} \frac{k_{x} k_{y}^{2}}{k_{z}^{3}}\right) y_{0},
\end{aligned}
$$

where we have again used the fact that $y_{1} \ll x_{1}$ if $a=1$. The second term is $-(e / m \gamma)^{2}\left(B_{w}^{2} / 2\right)\left(k_{y} / k_{z}^{2}\right) \times$ $\left(a^{2} k_{y} y_{0}+b^{2} k_{y} y_{0}\right)=-(e / m \gamma)^{2}\left(B_{w}^{2} / 2\right)\left(k_{y}^{2} / k_{z}^{2}\right) \times$ $\left(a^{2}+b^{2}\right) y_{0}$.

Putting term 1 and term 2 together we have

$$
\ddot{y}_{0}=-\left(\frac{e}{m \gamma}\right)^{2} \frac{B_{w}^{2}}{2} \frac{k_{y}^{2}}{k_{z}^{2}}\left(a^{2}+b^{2}\right) y_{0}\left(1-\frac{k_{x}^{2}}{k_{z}^{2}}\right)
$$

or, since $1-k_{x}^{2} / k_{z}^{2}=k_{y}^{2} / k_{z}^{2}$,

$$
\ddot{y}_{0}=-\left(\frac{e}{m \gamma}\right)^{2} \frac{B_{w}^{2}}{2} \frac{k_{y}^{2}}{k_{z}^{2}} \frac{k_{y}^{2}}{k_{z}^{2}}\left(a^{2}+b^{2}\right) y_{0} .
$$

The PPM and wiggler configurations have equal vertical focusing strengths. These are a factor of $k_{y}^{2} / k_{x}^{2}$ and $\left(k_{y}^{2} / k_{x}^{2}\right)^{2}$ stronger than the $x_{0}$ focusing strength for wiggler and PPM focusing, respectively. For emittance-dominated beams, this equation is all that is needed to understand the dynam- 
ics, and for space-charge dominated beams, the spacecharge force must be added.

Comparing these transverse equations of motion to the envelope equation, Eqs. (7) and (8), we recognize that balanced flow can be achieved in the vertical dimension if

$$
\left(\frac{e}{\beta c m \gamma}\right)^{2} \frac{B_{w}^{2}}{2} \frac{k_{y}^{4}}{k_{z}^{4}}=\frac{I / I_{A}}{\gamma^{3} \beta^{3}} \frac{1}{X+Y} \frac{1}{Y}+\frac{\varepsilon_{y, \text { norm }}^{2}}{Y^{4} \gamma^{2} \beta^{2}}
$$

for both wiggler and PPM focusing, and in the horizontal dimension if

$$
\left(\frac{e}{\beta c m \gamma}\right)^{2} \frac{B_{w}^{2}}{2} \frac{k_{x}^{2} k_{y}^{2}}{k_{z}^{2} k_{z}^{2}}=\frac{I / I_{A}}{\gamma^{3} \beta^{3}} \frac{1}{X+Y} \frac{1}{X}+\frac{\varepsilon_{x, \text { norm }}^{2}}{X^{4} \gamma^{2} \beta^{2}}
$$

for a wiggler-focused beam and if

$$
\left(\frac{e}{\beta c m \gamma}\right)^{2} \frac{B_{w}^{2}}{2} \frac{k_{x}^{4}}{k_{z}^{4}}=\frac{I / I_{A}}{\gamma^{3} \beta^{3}} \frac{1}{X+Y} \frac{1}{X}+\frac{\varepsilon_{x, \text { norm }}^{2}}{X^{4} \gamma^{2} \beta^{2}}
$$

for a PPM-focused beam.

The magnetic field required for equilibrium focusing an emittance-dominated beam is thus

$$
B_{\text {req }}=\sqrt{2} \varepsilon_{y, \text { norm }} \frac{m c}{Y^{2} e} \frac{k_{z}^{2}}{k_{y}^{2}} .
$$

Comparing this to Eq. (9), we see that the peak field required for equilibrium flow is larger when two-plane focusing is added, because of the field splitting between the vertical and horizontal dimensions. The field increase can be rewritten as $B_{\text {two-plane }}=B_{\text {single-plane }}\left[1+\left(k_{x}^{2} / k_{y}^{2}\right)\right]$. Now, the factor of $\frac{1}{2}$ multiplying the peak magnetic field in Eq. (23) came from both $\cos \left(k_{z} z\right)^{2}$ and $\sin \left(k_{z} z\right)^{2}$ integrations, which is different from the single-plane focusing case. Because $\cos \left(k_{z} z\right)^{2}+\sin \left(k_{z} z\right)^{2}$ is unity (so equal mixtures of these terms would not add to the Mathieu instability), we would expect that this mixture will change the Mathieu parameter somewhat. Inspecting the derivation closely, we infer that Mathieu parameter $\alpha$ is now

$$
\alpha=\frac{B_{w}^{2}}{2}\left(\frac{e}{m c \gamma \beta}\right)^{2}\left(\frac{L}{2 \pi}\right)^{2}\left(1-\frac{k_{x}^{4}}{k_{z}^{4}}\right),
$$

which is slightly different than $\alpha$ for single-plane focusing [Eq. (10)]. Expressed in terms of the beam emittance, we find

$$
\alpha=\frac{\varepsilon_{y, \text { norm }}^{2}}{\gamma^{2} \beta^{2}} \frac{1}{Y^{4}}\left(\frac{L}{2 \pi}\right)^{2}\left(\frac{k_{z}^{2}+k_{x}^{2}}{k_{z}^{2}-k_{x}^{2}}\right) .
$$

We conclude that the stability regions (in terms of the Mathieu stability regions) is a little worse with two-plane focusing (but only a little because $k_{x}^{2} \ll k_{z}^{2}$ ), so generally we expect that the stability analysis in [4] should nominally apply to two-plane focusing also. One of the key observations in [4] was that near the Mathieu stability limit, better beam transport is always found with a larger equilibrium beam radius, because at a smaller equilibrium beam radius [formed with a larger applied magnetic field and resulting in a larger Mathieu parameter, here given by Eq. (27)] the maximum beam envelope excursion will be much larger and will in fact exceed the maximum beam envelope excursion for the beam transport with the larger equilibrium radius for cases nearing the stability limit. Also, from Eq. (26) we find that as the beam is bunched and power is extracted, the required magnetic field for equilibrium transport is unchanged (as long as the transverse forces do not increase the normalized beam emittance). A consequence of these observations is that for emittancedominated sheet-beam transport in $\mathrm{rf}$ tubes, the peak applied magnetic field should not be larger than that required for equilibrium transport, unlike for space-charge dominated beams. Any increase in applied magnetic field from the equilibrium value decreases the equilibrium radius and brings the beam closer to the stability limit, leading to larger envelope ripple amplitudes.

\section{LONGITUDINAL VELOCITY MODULATIONS}

For good axial coherence of the beam, we want the longitudinal velocity to be constant during the $x_{0}$ betatron oscillation. The longitudinal velocity for natural focusing has been shown to be negligible for both wiggler and PPM configurations [4]. Additionally, Scharlemann has shown [8] that the transverse velocity $v_{\perp}$ is constant (wiggle averaged) over the betatron period, for wiggler focusing (which means that on average the longitudinal velocity is constant too, under the influence of only a magnetic field). We can show the same is true for PPM focusing too.

To show this, we will find $v_{\perp}^{2}=\dot{x}_{1}^{2}+\dot{y}_{1}^{2}+\dot{x}_{0}^{2}+\dot{y}_{0}^{2}$, wiggling averaging the fast motion terms. We will use

$$
\begin{aligned}
\dot{x}_{1}= & \frac{e}{m \gamma} B_{w} \frac{k_{y}}{k_{z}^{2}} \cosh \left(k_{x} x\right) \sin \left(k_{z} z\right)\left[a \cosh \left(k_{y} y\right)\right. \\
& \left.+b \sinh \left(k_{y} y\right)\right], \\
\dot{y}_{1}= & -\frac{e}{m \gamma} B_{w} \frac{k_{x}}{k_{z}^{2}} \sinh \left(k_{x} x\right) \sin \left(k_{z} z\right)\left[a \sinh \left(k_{y} y\right)\right. \\
& \left.+b \cosh \left(k_{y} y\right)\right]
\end{aligned}
$$

and write

$$
x_{0}=x_{\beta} \cos \left(k_{\beta x} z+\phi_{x}\right), \quad y_{0}=y_{\beta} \cos \left(k_{\beta y} z+\phi_{y}\right),
$$

where the betatron wave numbers are (from before):

$$
\begin{aligned}
k_{\beta x} & =\left(\frac{e}{m \gamma}\right) \frac{B_{w}}{\sqrt{2}} \frac{k_{x}}{k_{z}}\left(a \frac{k_{y}}{k_{z}}+b \frac{k_{x}}{k_{z}}\right), \\
k_{\beta y} & =\left(\frac{e}{m \gamma}\right) \frac{B_{w}}{\sqrt{2}} \frac{k_{y}^{2}}{k_{z}^{2}}(a+b) .
\end{aligned}
$$

Squaring the velocities and dropping the cross terms (as we have done before) we have 


$$
\begin{aligned}
& \overline{\dot{x}}_{1}^{2}=\left(\frac{e}{m \gamma}\right)^{2} \frac{B_{w}^{2}}{2} \frac{k_{y}^{2}}{k_{z}^{2}}\left[a^{2}\left(1+k_{x}^{2} x_{0}^{2}+k_{y}^{2} y_{0}^{2}\right)+b^{2} k_{y}^{2} y_{0}^{2}\right], \\
& \dot{y}_{1}^{2}=\left(\frac{e}{m \gamma}\right)^{2} \frac{B_{w}^{2}}{2} \frac{k_{x}^{2}}{k_{z}^{2}}\left[a^{2} k_{x}^{2} x_{0}^{2} k_{y}^{2} y_{0}^{2}+b^{2} k_{x}^{2} x_{0}^{2}\left(1+k_{y}^{2} y_{0}^{2}\right)\right]
\end{aligned}
$$

where the bars indicate an average over a focusing period, and

$$
\begin{aligned}
& \dot{x}_{0}^{2}=x_{\beta}^{2} \sin ^{2}(\bar{X})\left(\frac{e}{m \gamma}\right)^{2} \frac{B_{w}^{2}}{2} \frac{k_{x}^{2}}{k_{z}^{2}}\left(a^{2} \frac{k_{y}^{2}}{k_{z}^{2}}+b^{2} \frac{k_{x}^{2}}{k_{z}^{2}}\right) \dot{z}_{0}^{2}, \\
& \dot{y}_{0}^{2}=y_{\beta}^{2} \sin ^{2}(\bar{Y})\left(\frac{e}{m \gamma}\right)^{2} \frac{B_{w}^{2}}{2} \frac{k_{y}^{2}}{k_{z}^{2}}\left(a^{2}+b^{2}\right) \frac{k_{y}^{2}}{k_{z}^{2}} \dot{z}_{0}^{2},
\end{aligned}
$$

where we are using $\bar{X}=k_{\beta x} z+\phi_{x}$ and $\bar{Y}=k_{\beta y} z+\phi_{y}$.

The transverse velocity squared becomes

$$
\begin{aligned}
v_{\perp}^{2}= & \overline{\dot{x}}_{1}^{2}+\dot{y}_{1}^{2}+\dot{x}_{0}^{2}+\dot{y}_{0}^{2} \\
= & \left(\frac{e}{m \gamma}\right)^{2} \frac{B_{w}^{2}}{2} \dot{z}_{0}^{2}\left\{\frac { k _ { y } ^ { 2 } } { k _ { z } ^ { 4 } } \left[a^{2}\left(1+k_{y}^{2} y_{\beta}^{2} \cos ^{2} \bar{Y}+k_{x}^{2} x_{\beta}^{2} \cos ^{2} \bar{X}\right)\right.\right. \\
& \left.+b^{2} k_{y}^{2} y_{\beta}^{2} \cos ^{2} \bar{Y}\right]+\frac{k_{x}^{2}}{k_{z}^{4}}\left(a^{2} k_{y}^{2} y_{\beta}^{2} k_{x}^{2} x_{\beta}^{2} \cos ^{2} \bar{Y} \cos ^{2} \bar{X}\right. \\
& \left.+b^{2} k_{x}^{2} x_{\beta}^{2} \cos ^{2} \bar{X}\right)+x_{\beta}^{2} \sin ^{2}(\bar{X}) \frac{k_{x}^{2}}{k_{z}^{2}}\left(a^{2} \frac{k_{y}^{2}}{k_{z}^{2}}+b^{2} \frac{k_{x}^{2}}{k_{z}^{2}}\right) \\
& \left.+y_{\beta}^{2} \sin ^{2}(\bar{Y}) \frac{k_{y}^{2}}{k_{z}^{2}}\left(a^{2}+b^{2}\right) \frac{k_{y}^{2}}{k_{z}^{2}}\right\}
\end{aligned}
$$

or

$$
\begin{aligned}
v_{\perp}^{2}= & \left(\frac{e}{m \gamma}\right)^{2} \frac{B_{w}^{2}}{2} \dot{z}_{0}^{2} \frac{1}{k_{z}^{4}}\left\{a ^ { 2 } k _ { y } ^ { 2 } \left(k_{y}^{2} y_{\beta}^{2} \cos ^{2} \bar{Y}+k_{x}^{2} x_{\beta}^{2} \cos ^{2} \bar{X}\right.\right. \\
& \left.+k_{y}^{2} y_{\beta}^{2} \sin ^{2} \bar{Y}+k_{x}^{2} x_{\beta}^{2} \sin ^{2} \bar{X}\right) \\
& +a^{2} k_{x}^{4} k_{y}^{2} x_{\beta}^{2} y_{\beta}^{2} \cos ^{2} \bar{X} \cos ^{2} \bar{Y}+b^{2}\left(k_{y}^{4} y_{\beta}^{2} \cos ^{2} \bar{Y}\right. \\
& \left.\left.+k_{x}^{4} x_{\beta}^{2} \cos ^{2} \bar{X}+k_{y}^{4} y_{\beta}^{2} \sin ^{2} \bar{Y}+k_{x}^{4} x_{\beta}^{2} \sin ^{2} \bar{X}\right)\right\}
\end{aligned}
$$

and we are left with

$$
\begin{aligned}
v_{\perp}^{2}= & \left(\frac{e}{m \gamma}\right)^{2} \frac{B_{w}^{2}}{2} \dot{z}_{0}^{2} \frac{1}{k_{z}^{4}}\left\{a^{2} k_{y}^{2}\left(k_{y}^{2} y_{\beta}^{2}+k_{x}^{2} x_{\beta}^{2}\right)+b^{2}\left(k_{y}^{4} y_{\beta}^{2}\right.\right. \\
& \left.\left.+k_{x}^{4} x_{\beta}^{2}\right)\right\} .
\end{aligned}
$$

The transverse and the longitudinal velocities are constant with wiggle averaging for both wiggler and PPM fields to first order. This is important for coherent amplification - the bunches formed under the influence of the rf stay bunched. Note that $v_{\perp}^{2}$ is bigger for wiggler focusing than PPM focusing by a factor of $k_{y} / k_{x}$.

\section{NUMERICAL ANALYSIS OF STABILITY WITH TWO-PLANE FOCUSING-LOW-EMITTANCE CASE}

The purpose of this section is to give a quantitative comparison between two-plane focusing with a wiggler configuration and a PPM configuration. We will see that two-plane wiggler focusing has similar stability to natural wiggler focusing, but two-plane PPM focusing is greatly degraded from natural PPM focusing.

It has been pointed out in [9] that it may not be practical to produce enough horizontal focusing for some cases with two-plane PPM focusing. From Eq. (9), we see that the vertical focusing for an emittance-dominated beam is independent of beam energy, but the defocusing spacecharge force decreases with increasing energy. For a sufficiently high energy beam, it is possible to generate large enough transverse magnetic fields for equilibrium focusing with a two-plane PPM configuration.

Equations (23)-(25) give us the ability to estimate the amount of two-plane focusing required for stable flow. The simulations in this section include all beam effects - space charge and emittance effects in both the horizontal and vertical dimensions. In Fig. 3, we plotted the horizontal beam-size expansion with just natural focusing. The beam expands to about $3 \mathrm{~cm}$ over a $10 \mathrm{~cm}$ drift. The first thing we need to do is to determine how much horizontal focusing we need to confine the beam.

In the Sec. I, we showed that for the nominal beam parameters of a $10 \mathrm{~mm}$ by $0.5 \mathrm{~mm}$ beam, with $Y=$ $0.125 \mathrm{~mm}$ and $X=2.5 \mathrm{~mm}$, the space-charge term is $1.18 \mathrm{~m}^{-1}$ (both horizontal and vertical envelope equation), and the emittance term is $1.79 \mathrm{~m}^{-1}$ for the vertical envelope equation (and $8.9 \times 10^{-4} \mathrm{~m}^{-1}$ for the horizontal envelope equation), and that a peak magnetic field of $0.266 \mathrm{~T}$ is needed to vertically focus the beam with natural focusing. Using Eqs. (23)-(25), we find that $k_{x} / k_{y}=0.14$, $k_{y} / k_{z}=0.99$, and $k_{x} / k_{z}=0.138$ for matched two-plane focusing with wiggler focusing, and that the peak magnetic field must be $0.268 \mathrm{~T}$. Likewise, we find that $k_{x} / k_{y}=$ $0.375, k_{y} / k_{z}=0.936$, and $k_{x} / k_{z}=0.351$ for matched two-plane focusing with PPM focusing, and that the peak magnetic field must be $0.284 \mathrm{~T}$.

For wiggler focusing, the best transport was found with a slightly different peak field $(0.263 \mathrm{~T})$, but with the proper ratio of the focusing wave numbers $\left(k_{x} / k_{y}=0.14\right)$. The transport is shown in Fig. 4.

The slight variation in peak field is due to the phase of the entrance condition of the beam relative to the focusing period (the beam is injected at a vertical width of $0.5 \mathrm{~mm}$, but depending on the phase of the orbit, the actual equilibrium radius can be the same, larger, or smaller). This variation is typically on the order of a few percent.

The peak magnetic field needed for equilibrium flow with PPM focusing is numerically found to be about $0.2725 \mathrm{~T}$ (shown in Fig. 5 with natural focusing and where the horizontal space-charge force has been suppressed). The slight different field $(0.2725 \mathrm{~T}$ instead of $0.284 \mathrm{~T})$ is due to the starting phase of the oscillation.

In Fig. 6 we show the calculated flow for the nominal high-aspect-ratio beam with a peak field of $0.291 \mathrm{~T}$ and a 


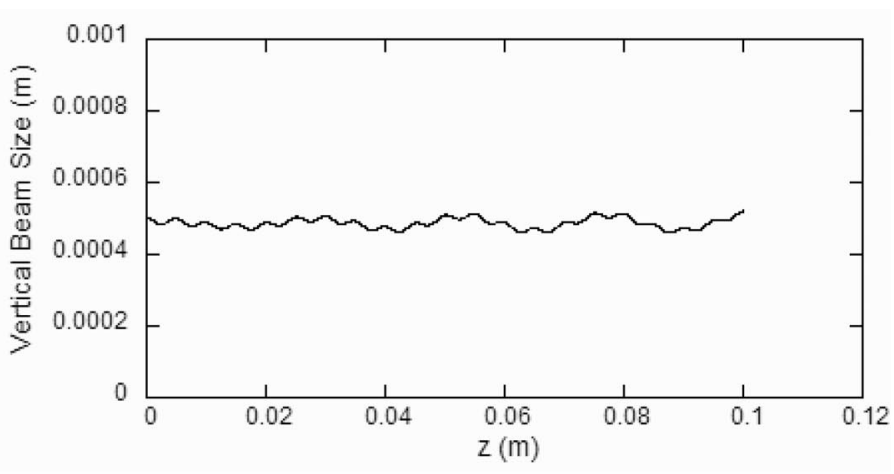

(a)

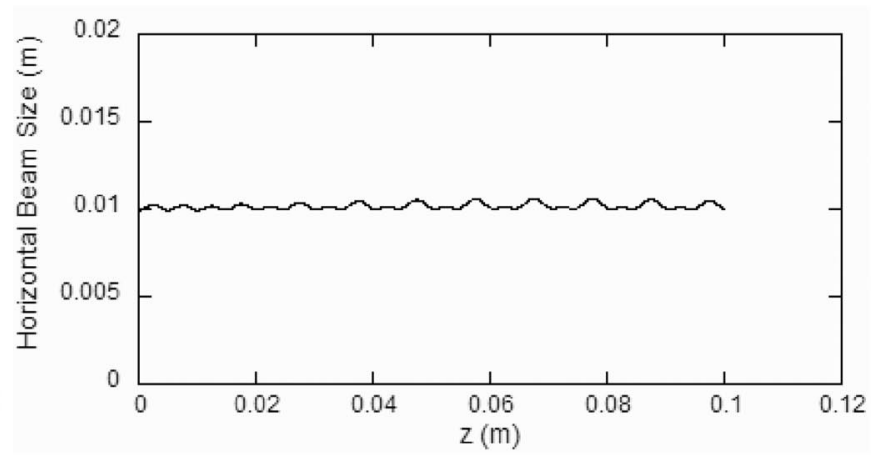

(b)

FIG. 4. Nominal two-plane focusing case for wiggler focusing, with peak field of $0.263 \mathrm{~T}$ and a wave number ratio of 0.14 . (a) Vertical beam size versus axial position. (b) Horizontal beam size versus axial position.

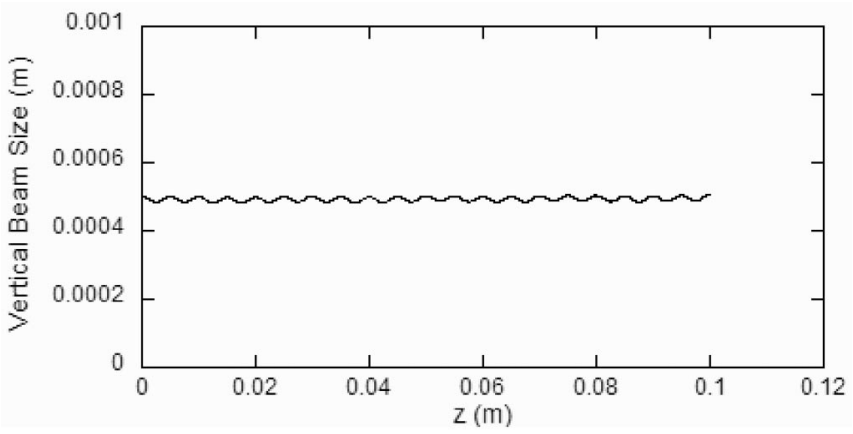

FIG. 5. Natural focusing with both vertical space-charge and emittance effects for PPM focusing. Balanced flow is achieved with a peak field of about $0.2725 \mathrm{~T}$.

wave number ratio of $k_{x} / k_{y}=0.375$ (numerically found to give the best possible transport), with full transverse dynamics. The transport is very poor, and significantly worse than that with wiggler focusing.

The poor focusing is due to fact that for a $1-\mathrm{cm}$ wide beam, the term $k_{x} x$ is greater than 2 (for PPM focusing), which introduces large nonlinear focusing forces, exacerbated by the fact that $y$ can significantly increase due to the PPM flutter motion. This can be easily seen in the final $x-y$ beam distribution, shown in Fig. 7. The beam distribution has even folded over a couple of times.

In order to investigate this phenomena better, we next reduce the horizontal beam space-charge force by a factor of 26, which lets us use a wave number ratio of $k_{x} / k_{y}=$ 0.14 , as was used for the wiggler transport. The transport is significantly better, and shown in Fig. 8.

Even with the reduced wave number, though, there still are enough nonlinear terms to cause distortion of the beam. This indicates that the vertical flutter motion of the beam (absent for wiggler focusing) is contributing to the nonlinearities.

In Fig. 9, we compare the normalized envelope amplitude versus focusing period with two-plane wiggler focusing to that with the same vertical effects but suppressing the horizontal space-charge force. Some minor degradation of the equilibrium is seen, and the stability threshold is slightly decreased, for a transverse wave number ratio of

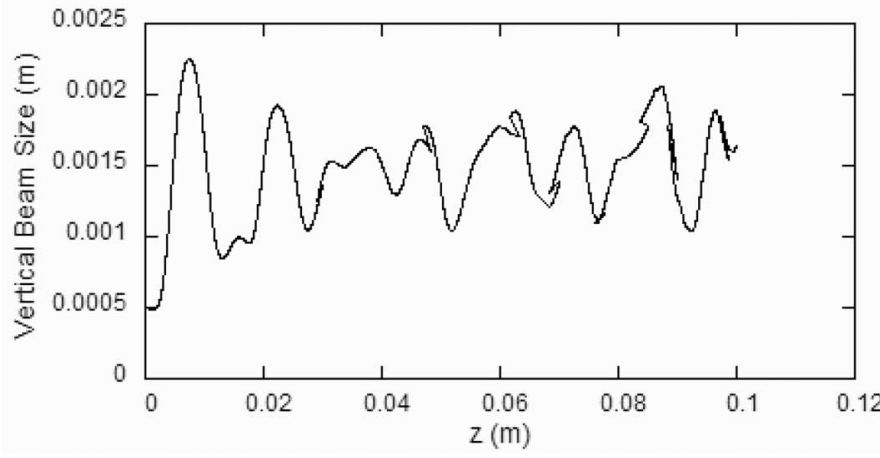

(a)

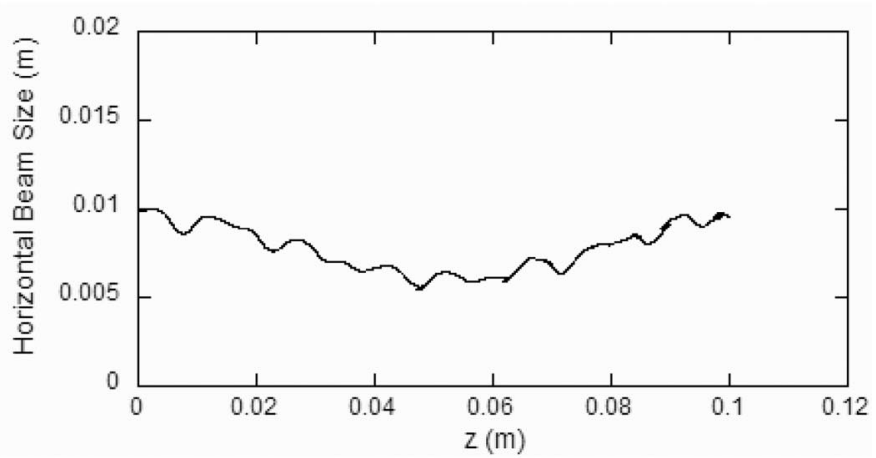

(b)

FIG. 6. Nominal two-plane focusing case for PPM focusing, with peak field of $0.291 \mathrm{~T}$ and a wave number ratio of 0.375 . (a) Vertical beam size versus axial position. (b) Horizontal beam size versus axial position. 


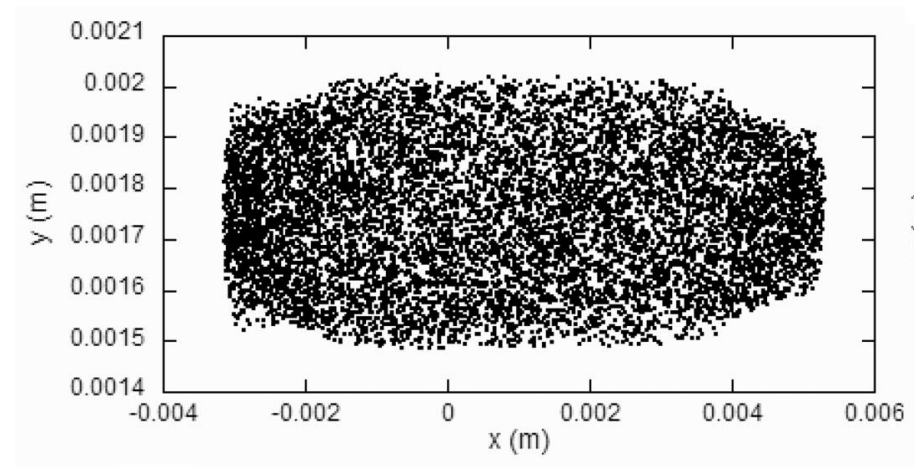

(a)

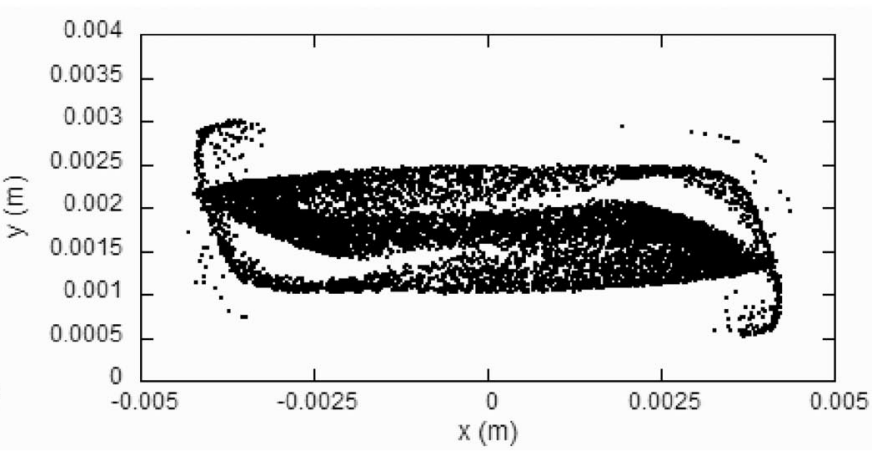

(b)

FIG. 7. Final $x-y$ beam distribution for (a) the nominal wiggler focusing case and (b) the nominal PPM focusing case.

0.14 . It should be noted that as the period of the focusing increased, the effective vertical focusing is also increased, because of the nonparaxial horizontal beam motion [4]. As a result, the peak magnetic field in these simulations was decreased to maintain the best equilibrium flow (by about $15 \%$ at a $2.75-\mathrm{cm}$ focusing period).

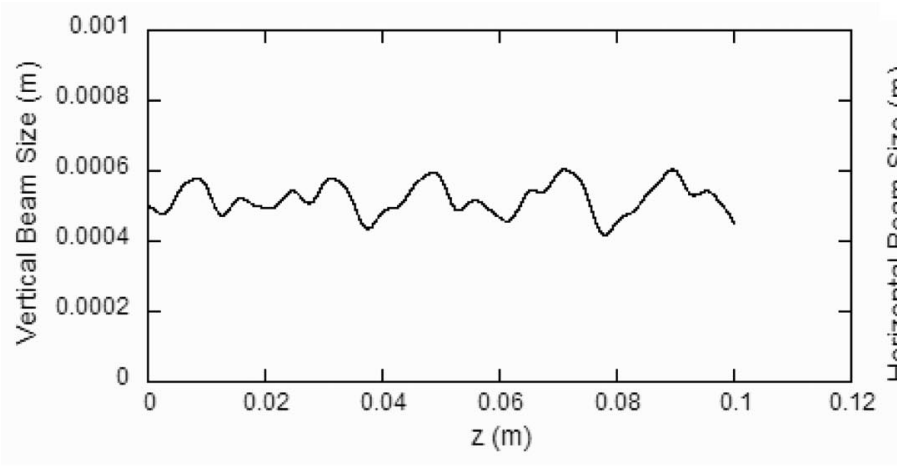

(a)

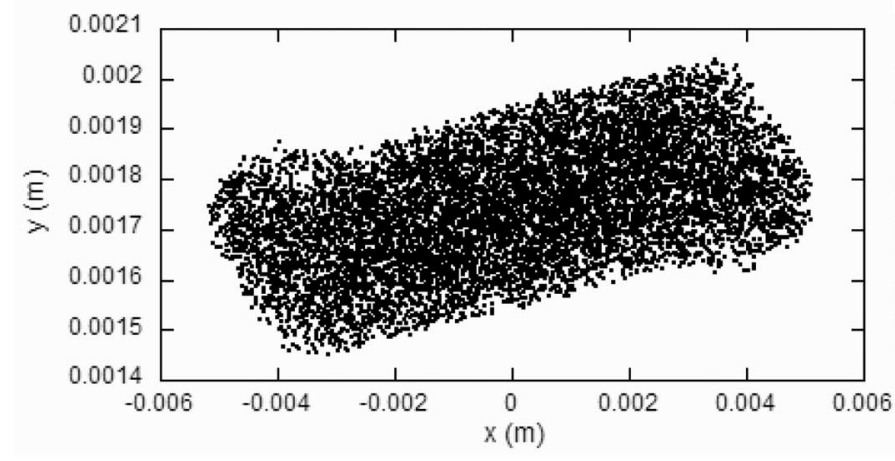

(c)

\section{COMPARISON OF DIFFERENT HORIZONTAL FOCUSING SCHEMES-HIGH-EMITTANCE CASE}

An alternative to two-plane focusing of a sheet beam of the form shown in Eq. (3) is to have natural focusing

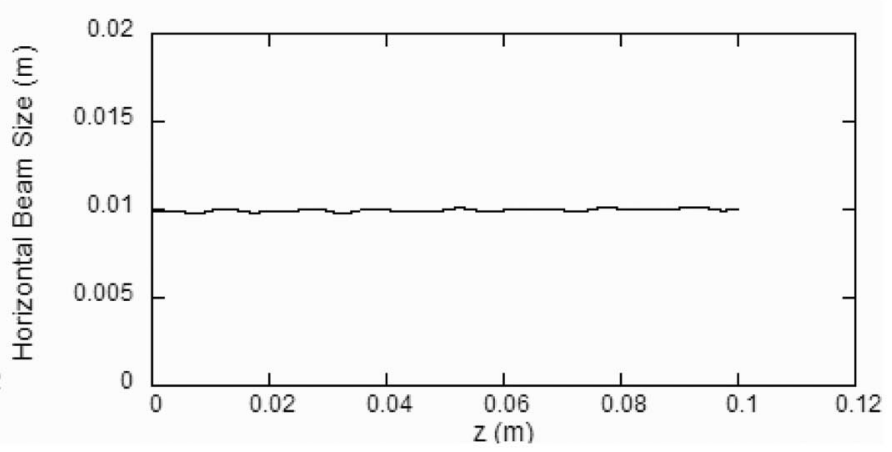

(b)

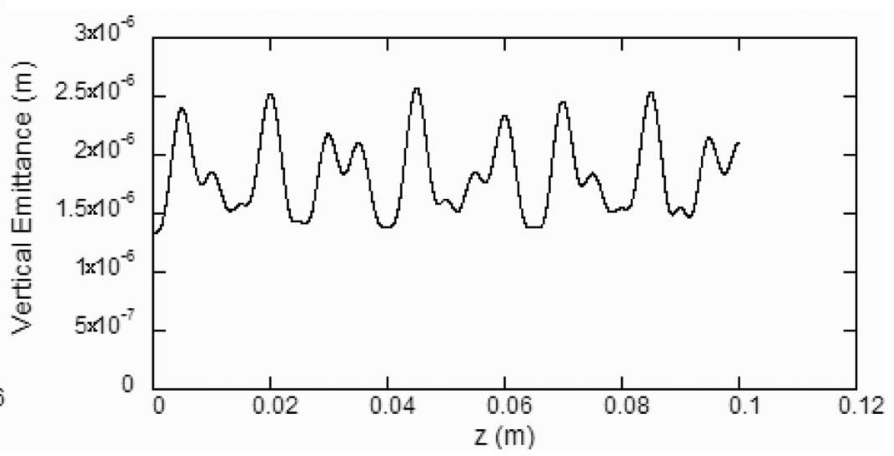

(d)

FIG. 8. Two-plane focusing case for PPM focusing with reduced horizontal space-charge force, with peak field of $0.295 \mathrm{~T}$ and a wave number ratio of 0.14 . (a) Vertical beam size versus axial position. (b) Horizontal beam size versus axial position. (c) Final $x-y$ beam distribution. (d) Vertical emittance versus axial position. 


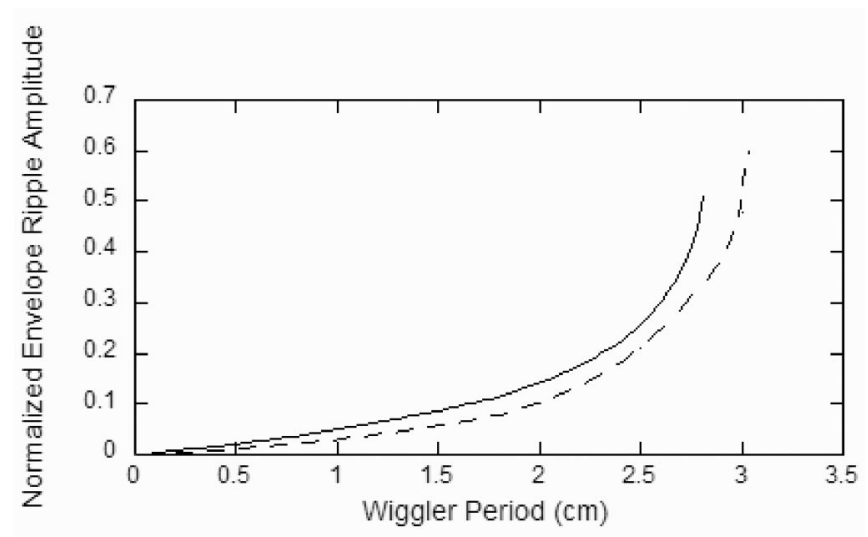

FIG. 9. Envelope ripple amplitude versus focusing period for wiggler focusing, for two-plane focusing (solid line) and for single-plane focusing (dashed line), both with vertical emittance and space-charge effects.

augmented by linear focusing introduced by a quadrupole field, where the added field is of the form

$$
\begin{aligned}
& B_{x}=y B_{0}^{\prime} \cos \left(k_{q} z\right), \quad B_{y}=x B_{0}^{\prime} \cos \left(k_{q} z\right), \\
& B_{z}=-x y k_{q} B_{0}^{\prime} \sin \left(k_{q} z\right),
\end{aligned}
$$

where $k_{q}$ is $2 \pi$ divided by the period of the quadrupole field. Note that if the field is axially constant $\left(k_{q}=0\right)$, the axial field vanishes. It is well known that an axially constant quadrupole field produces linear forces, although it is also known that a quadrupole field in a constant axial field of a solenoid can cause significant emittance growth [12]. The PPM field looks like an alternating solenoid, so there is a potential emittance growth mechanism, which we numerically quantify here. For the parameters studied here, this emittance growth is negligible, but this may be a concern for other types of beams.

The field shown in Eq. (37) is the lowest magnetic field multiple that occurs by either the addition of a large external quadrupole, by offsetting the wiggler magnets as in [9], notching the wiggler magnets as in $[10,11]$, or by adding nonsymmetric quadrupole focusing to the wiggler or PPM array as in [6,9]. Nonsymmetric configurations will induce additional magnetic multiples in addition to the field shown in Eq. (37), which will increase the beam emittance for the same amount of horizontal confinement. As a result, we will limit our study to field augmentation of the form shown in Eq. (37), with the understanding that this is the ideal field augmentation (in terms of minimizing beam emittance growth), and that physical realizations of this field may include high-order multiples which are not desirable. It should also be noted in terms of comparing practical designs that a somewhat higher PPM field is possible than a wiggler field, for the same period and same magnet field strength.

Previous work simulating the effect of quadrupole augmentation of PPM focusing [6,9] studied transport of space-charge dominated beams, mostly for short PPM periods well below the Mathieu limit. It was found that (i) an elliptical beam can be matched into such a two-plane focusing configuration but a rectangular cross-section beam cannot, (ii) the maximum period of the PPM structure with a uniform quadrupole field augmentation is consistent with the Mathieu limit, and (iii) for short periods, the PPM augmentation with a periodic quadrupole field is somewhat better than augmentation with a uniform field, due to a horizontal beam mismatch (leading to oscillations in the horizontal beam size) for the case simulated. As shown in [9], the space-charge field from a rectangularshaped beam is nonlinear, so it is not surprising that a space-charge dominated beam (with nonlinear horizontal defocusing forces) cannot be matched well by horizontal quadrupole focusing (with linear horizontal restoring forces). From Figs. 1(a), 1(b), and 9, we anticipate verifying that in the emittance regime that the transport stability of the quadrupole-augmented PPM structure is also consistent with the Mathieu stability and that the stability of the two-plane wiggler structure is somewhat less, due to the nonparaxial horizontal beam motion in the wiggler field [4].

Here we compare the transport of a relatively high emittance beam (normalized rms emittance of $4 \mu \mathrm{m}$, with other beam parameters the same as before), using two-plane wiggler focusing, to natural focusing augmented by a quadrupole field applied external to the PPM structure. In order to achieve somewhat more stability, the vertical equilibrium beam size was allowed to grow larger than $0.5 \mathrm{~mm}$. The initial beam size was assumed to be $0.6 \mathrm{~mm}$, and the phase of the betatron oscillation was numerically adjusted so the betatron oscillation was at a local maximum at particle injection. Because natural wiggler focusing is inferior to natural PPM focusing, wiggler focusing augmented by quadrupole fields was not studied.

We see in the following simulations that two-plane wiggler focusing is roughly equivalent to single-plane PPM focusing augmented with a constant quadrupole field, whereas single-plane PPM focusing augmented with a periodic quadrupole field is inferior to both other options.

\section{A. Baseline two-plane wiggler focusing transport}

This case is similar to the one in Sec. IV, but with higher peak field and a lower ratio of transverse to vertical focusing (because the vertical defocusing is larger with the greater emittance, but the horizontal space-charge force is the same). From Eq. (9), the equilibrium field for a 0.6$\mathrm{mm}$ wide beam is $0.43 \mathrm{~T}$. Numerically, equilibrium transport is found with a $0.40 \mathrm{~T}$ peak field and with $k_{x} / k_{y}=$ 0.090 . The vertical beam size is plotted versus axial position in Fig. 10, for wiggler periods from 8 to $16 \mathrm{~mm}$. The horizontal beam size is very constant, and there is no appreciable emittance growth for the 14-mm period case, shown in Fig. 11, which would be an acceptable design 

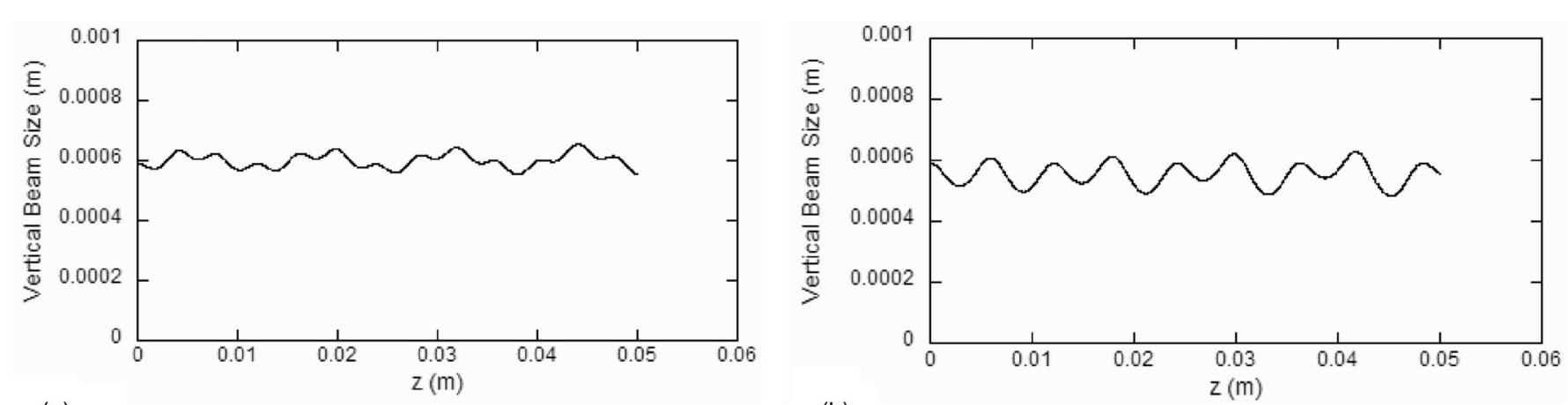

(a)

(b)

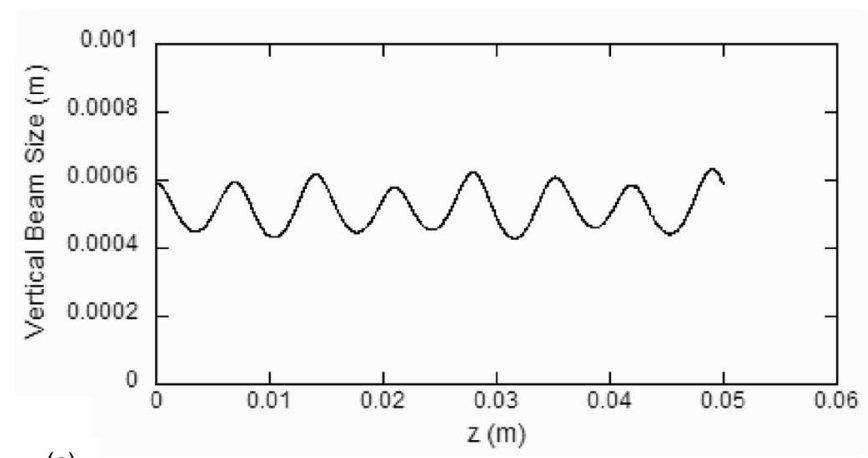

(c)
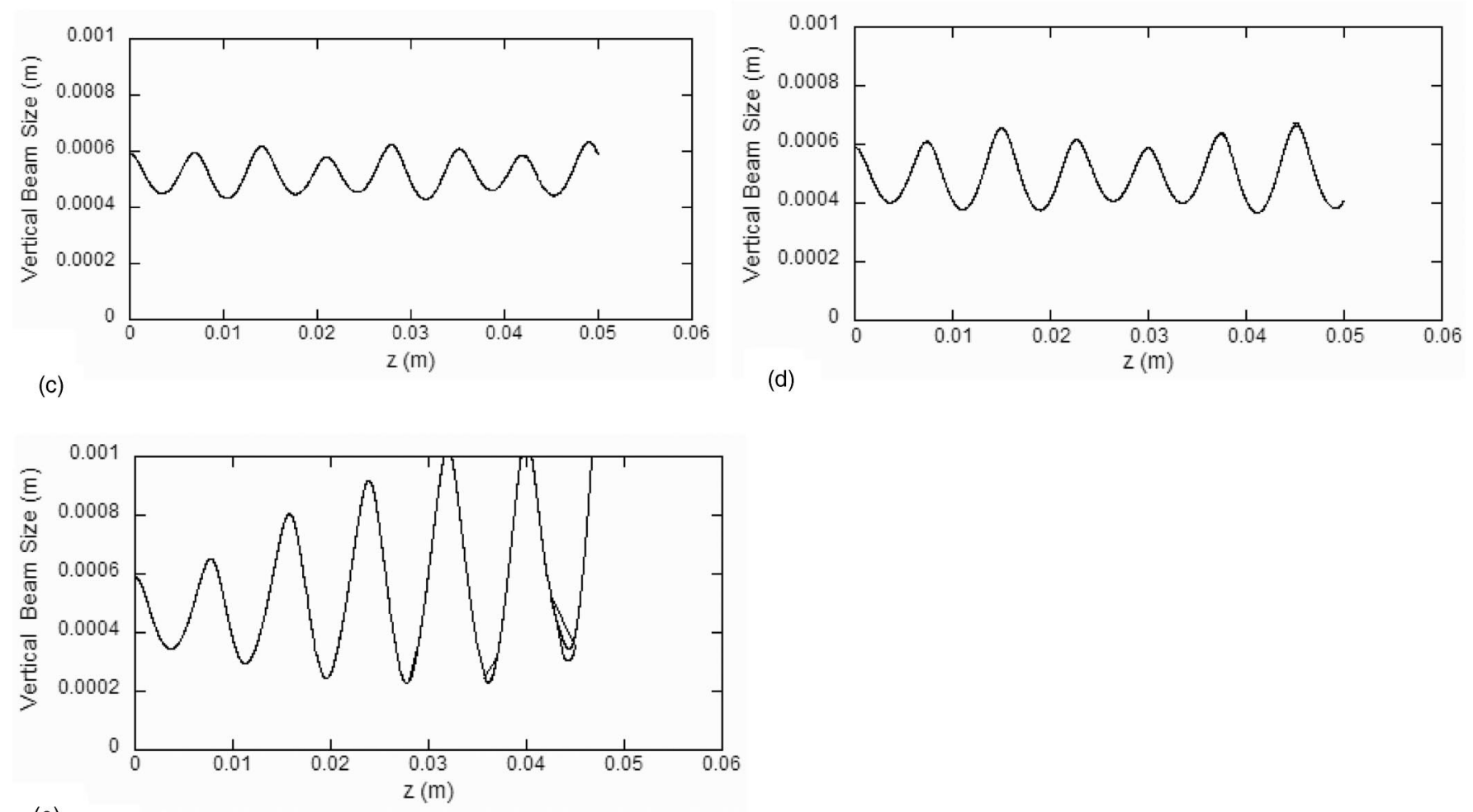

(e)

FIG. 10. Vertical beam size for two-plane wiggler focusing, high-emittance case (a) 8-mm period, (b) 12-mm period, (c) 14-mm period, (d) 15-mm period, (e) 16-mm period.

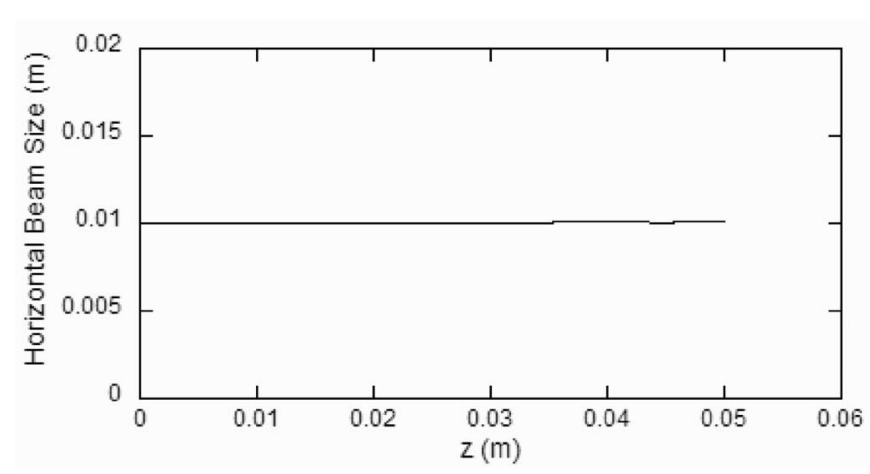

(a)

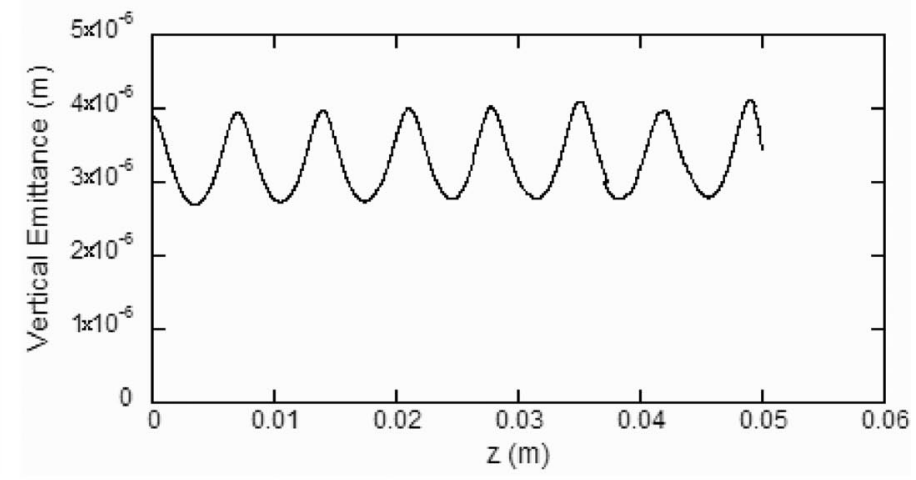

(b)

FIG. 11. (a) Horizontal beam size and (b) vertical emittance for a 14-mm period. 
point. From Figs. 10(d) and 10(e) it is observed that the stability limit for this combination of parameters is reached with a wiggler period of about $15 \mathrm{~mm}$. From Eq. (27), this corresponds to a limiting value of the Mathieu parameter $\alpha$ equal to 0.31 , which is consistent with the stability limit predicted in Fig. 1(a).

\section{B. Natural PPM focusing augmented by a constant quadrupole field}

The external quadrupole field focuses horizontally, but defocuses vertically. As a result, the peak PPM field needs

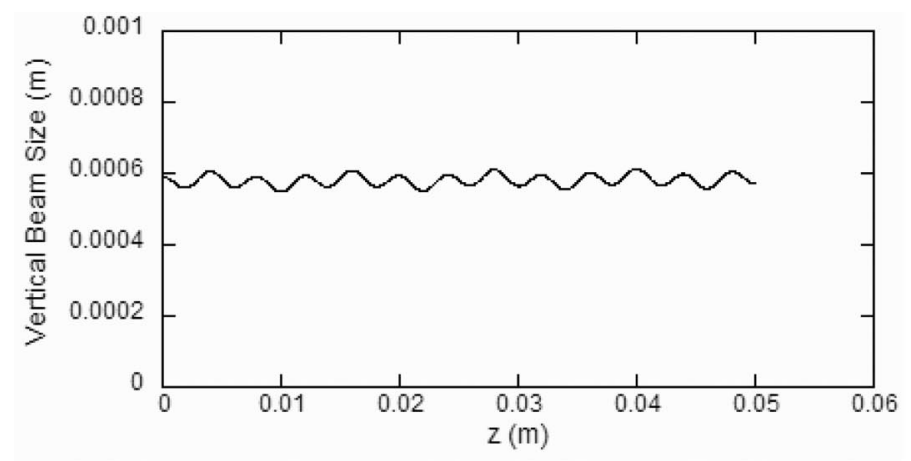

(a)

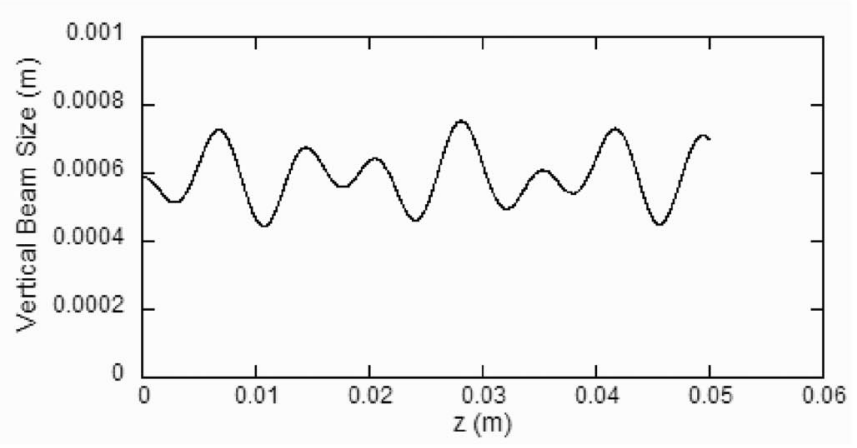

(c)

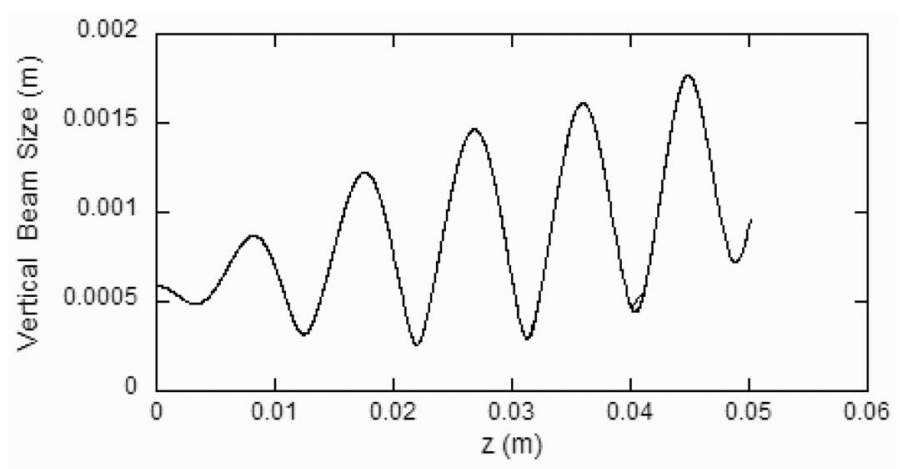

(e)

FIG. 12. Vertical beam size for PPM focusing augmented by uniform quadrupole, high-emittance case (a) 8-mm period, (b) 12-mm period, (c) 14-mm period, (d) 16-mm period, and (e) 18-mm period. to be higher than in the wiggler case in the previous part. Equilibrium flow is found with a peak PPM field of approximately $0.475 \mathrm{~T}$ and a quadrupole field of $0.59 \mathrm{~T} / \mathrm{m}$. In Fig. 12, we plot the vertical beam size as a function of axial position, for PPM periods of 8 to $18 \mathrm{~mm}$. In Fig. 13, we plot the horizontal beam size and vertical emittance for the 14$\mathrm{mm}$ period case, which is roughly comparable to the 14$\mathrm{mm}$ period wiggler case in terms of overall beam envelope scallop. The observed stability threshold at a period of about $17 \mathrm{~mm}$ in Fig. 12 correspond to a limiting value of the Mathieu parameter $\alpha$ of about 0.54 . We note that there

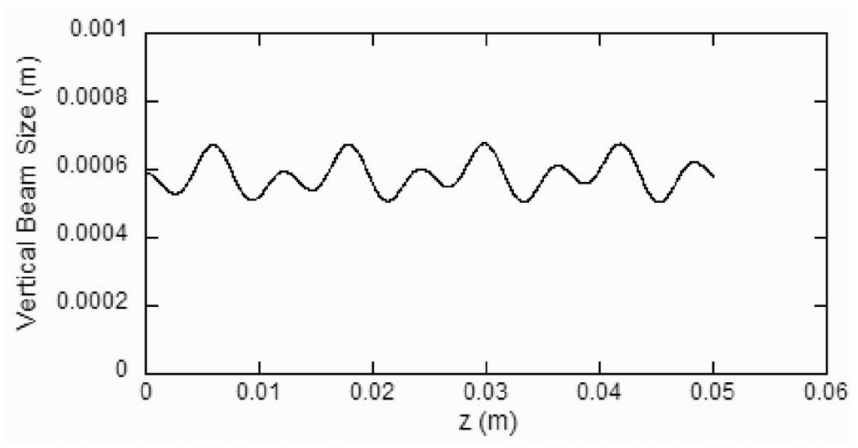

(b)

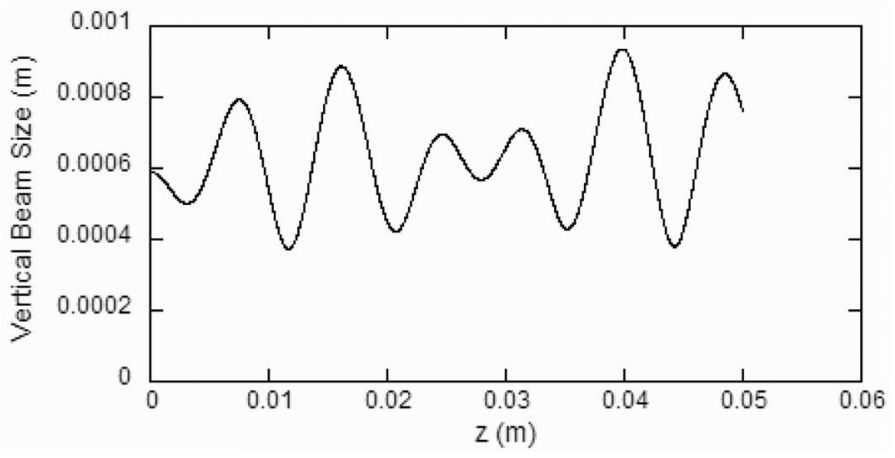

(d) 


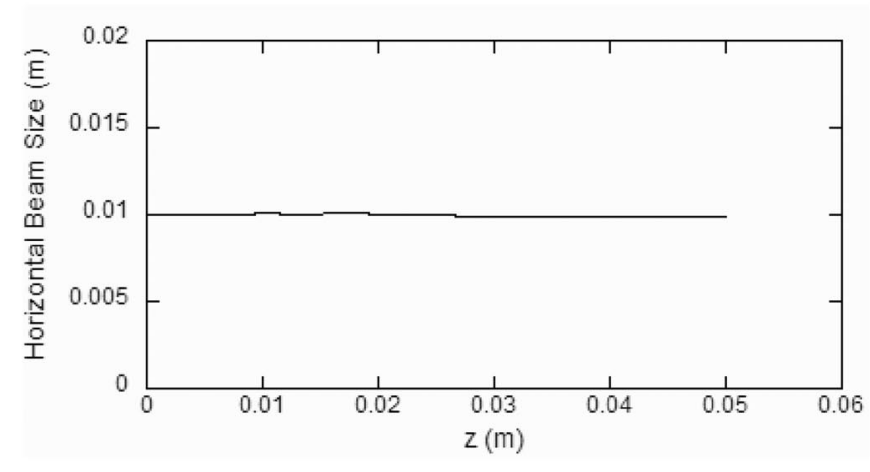

(a)

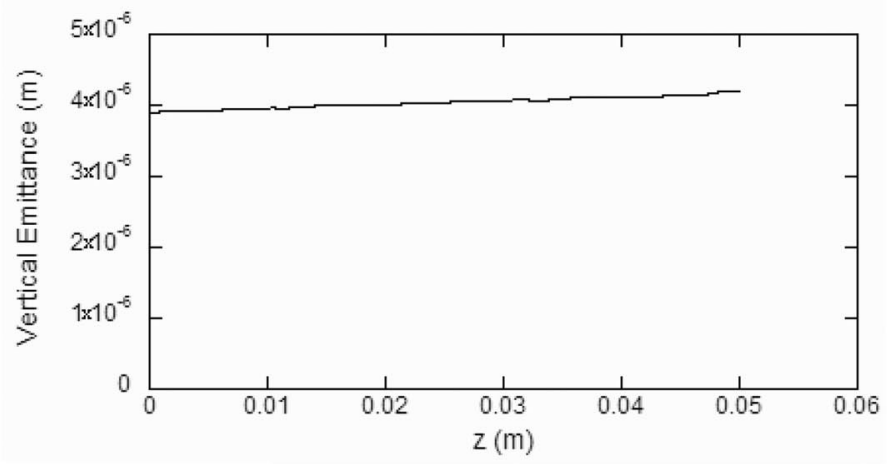

(b)

FIG. 13. (a) Horizontal beam size and (b) vertical emittance for a $14 \mathrm{~mm}$ period.

is a slight decrease in stability from the Mathieu limit of 0.66 (using the limit $\alpha=0.66$, the transport should be stable for focusing periods less that about $18.8 \mathrm{~mm}$ ), but overall, the simulations in Fig. 12 are mostly consistent with that shown in Fig. 1(b).

\section{Natural PPM focusing augmented by a periodic quadrupole field}

A uniform external quadrupole surrounding a PPM stack will tend to be relatively large physically. It has been proposed [6] to alternatively add quadrupole magnets to the PPM stack, and produce a periodic quadrupole field of the form in Eq. (37), where the quadrupole period is equal to the PPM period, $k_{q}=k_{z}$. Because the quadrupole field is periodic, the focusing is of second order, and the quadrupole strength must be increased as the period is decreased to maintain an equal amount of horizontal focusing. For the PPM periods analyzed, the peak quadrupole field is $60.0 \mathrm{~T} / \mathrm{m}$ for a $4-\mathrm{mm}$ period, $40.6 \mathrm{~T} / \mathrm{m}$ for a 6 $\mathrm{mm}$ period, $30.6 \mathrm{~T} / \mathrm{m}$ for a $8-\mathrm{mm}$ period, $24.3 \mathrm{~T} / \mathrm{m}$ for a $10-\mathrm{mm}$ period, and $21.0 \mathrm{~T} / \mathrm{m}$ for a $12-\mathrm{mm}$ period. The vertical beam size versus axial position is shown in Fig. 14 for these periods. The horizontal beam size versus axial position is shown in Fig. 15 and the vertical emittance for a nominal case (8-mm period) is shown in Fig. 16. The peak PPM field was also $0.475 \mathrm{~T}$ for these simulations. No emittance growth is seen due to the coupling of the axial and quadrupole fields (probably due to the small periodic angular beam rotation). However, much poorer transport stability is seen than previously reported in [6], probably because the case studied there had a very short period and low field strength (the emittance was assumed to be zero), which leads to a very small Mathieu parameter.

\section{DISCUSSION}

Our major conclusions are (i) with two-plane focusing, emittance-dominated beams have larger envelope scallop- ing than space-charge dominated beams, (ii) although PPM focusing is clearly superior for single-plane focusing, twoplane PPM focusing is unacceptable for wide elliptical beams, (iii) single-plane PPM focusing augmented by a uniform quadrupole focusing is roughly equivalent to twoplane wiggler focusing, and (iv) single-plane PPM focusing augmented by periodic quadrupole focusing has a greatly degraded stability threshold, because of the firstorder cancellation of the horizontally focusing quadrupole fields. The limiting factor for both two-plane wiggler focusing and quadrupole-augmented natural PPM focusing is a Mathieu-type stability threshold due to the length of the focusing period instead of actual emittance growth.

We see from Eq. (15), where the vertical betatron motion is given by $y_{1}=\left(e / m \gamma \dot{z}_{0}\right) B_{w}\left(k_{x}^{2} / k_{z}^{3}\right) x \cos \left(k_{z} z\right)\left(a k_{y} y+b\right)$, the high-order PPM flutter motion $(a=0, b=1$ for PPM motion). The combination of weaker horizontal focusing with the same $k_{x}$ for the PPM field configuration (which in turns requires $k_{x}$ to be larger) plus the larger vertical beam movement leads to large arguments in the sinh functions in the vertical force term, which in turn leads to significant vertical emittance growth.

We see from Sec. V that the transport stability of a highemittance beam in a two-plane wiggler structure and in a PPM structure augmented by a uniform quadrupole is mostly predicted by the Mathieu stability parameter, in agreement with previous analyses. More precisely, we have seen about a $10 \%$ decrease in the maximum focusing period as compared with just natural focusing. However, we see that the transport stability of a high-emittance beam in a PPM structure augmented by a periodic quadrupole is greatly degraded as the focusing period is even moderately increased. Numerically, we were able to easily horizontally match a high-emittance beam into a PPM structure augmented with a uniform quadrupole structure. We suspect the horizontal beam-size oscillations seen in [6] were due to difficulties in tuning the quadrupole field strength separately from the natural focusing, as the quadrupole field was not added in separately but was included with the PPM structure as part of a magnetics simulation. 


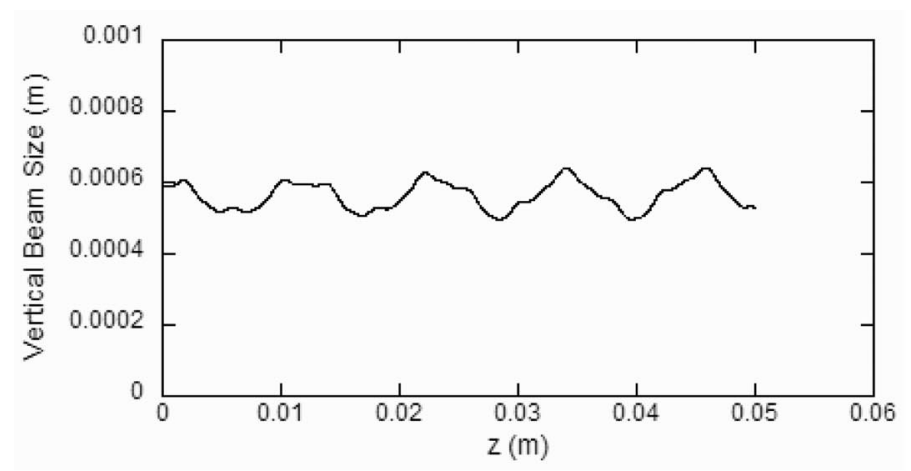

(a)

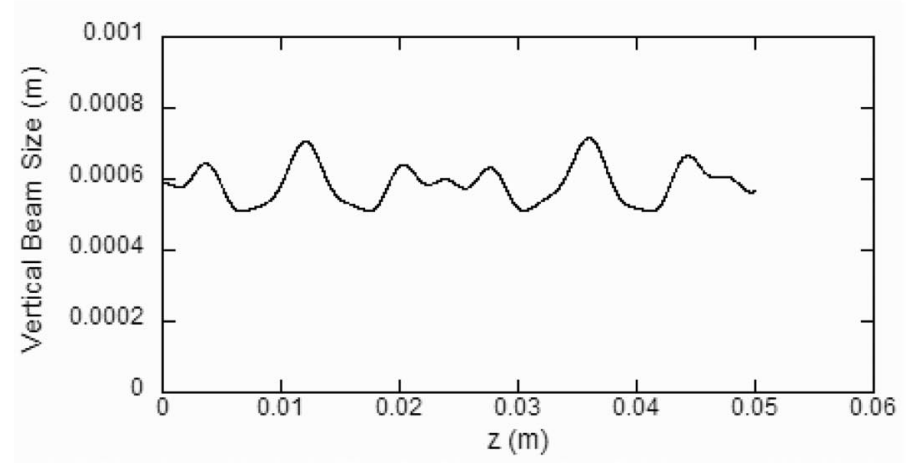

(c)

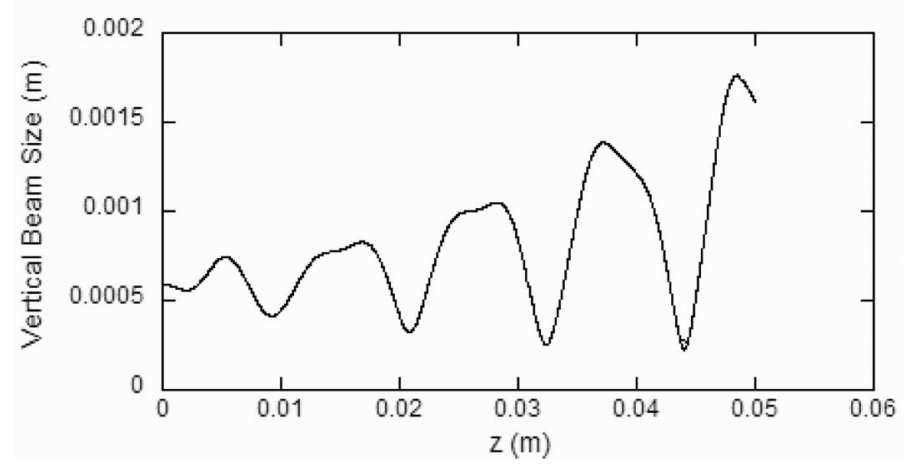

(e)

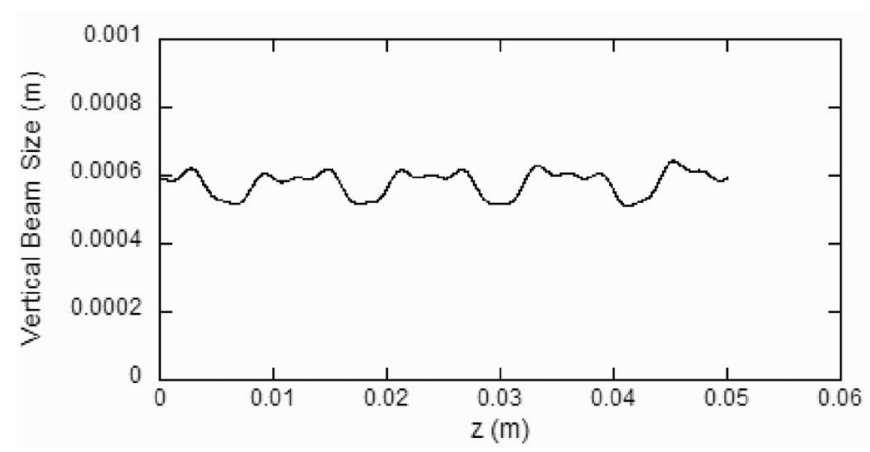

(b)

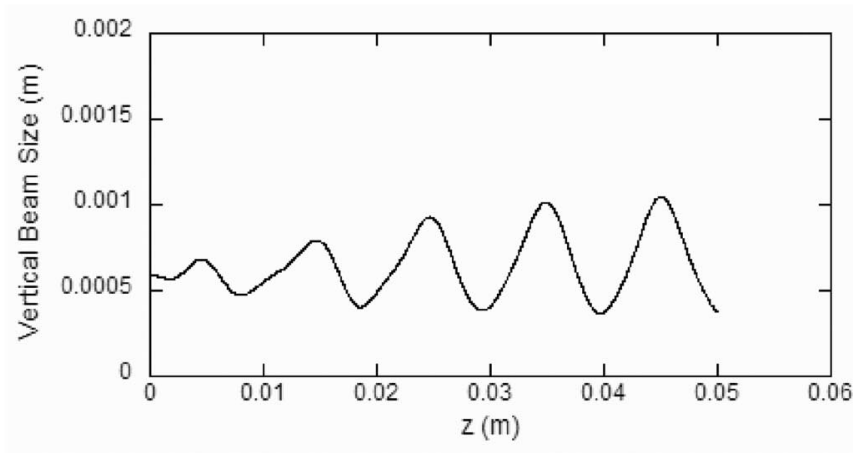

(d)

FIG. 14. Vertical beam size for PPM focusing augmented by periodic quadrupole (a) 4-mm period, (b) 6-mm period, (c) 8-mm period, (d) 10-mm period, and (e) 12-mm period.

For the case of natural PPM focusing augmented with a constant quadrupole field, the vertical defocusing effect of the quadrupole field requires a larger PPM field (about 20\% larger than the two-plane wiggler field). Mathieu-type instability is reached with a period about $10 \%$ greater than for two-plane wiggler focusing, but with roughly equivalent envelope scalloping for the same focusing period. One key design issue for practical devices is getting a large enough field (wiggler or PPM) in the beam gap, due to both the size of the beam gap and the size of individual magnets. As the period is increased, a larger field is possible, and for the Los Alamos case, the advantage of the weaker required field with two-plane wiggler focusing is essentially canceled by the disadvantage of the shorter focusing period. In addition, for the same gap size, the PPM 


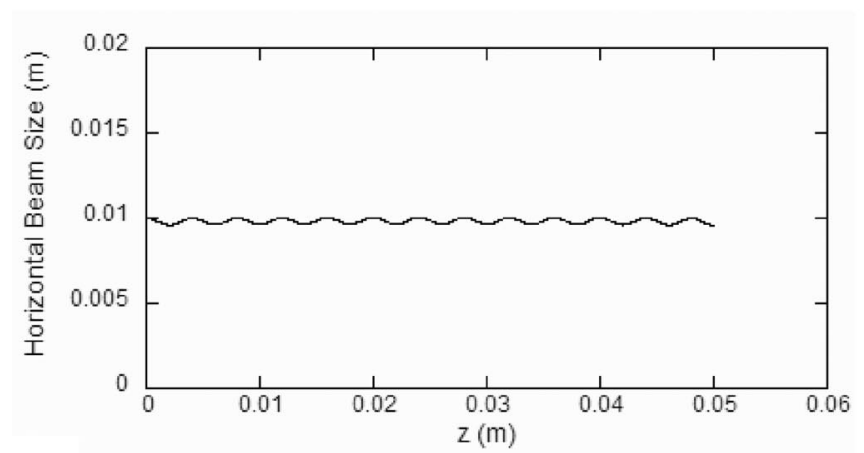

(a)

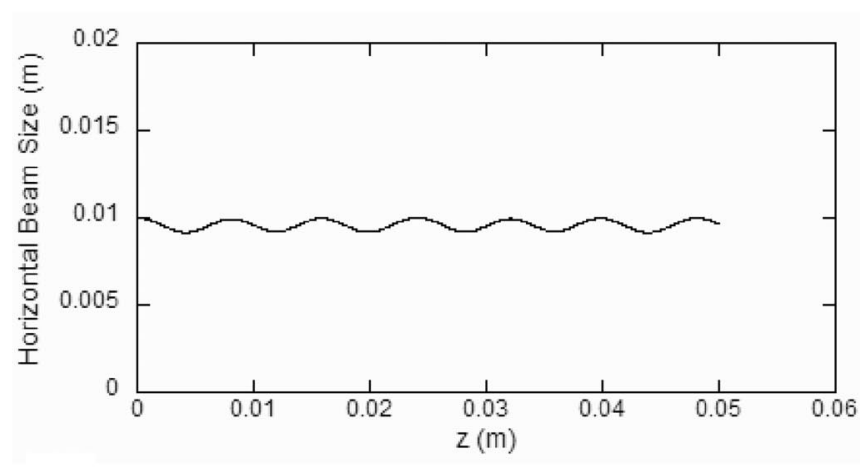

(c)

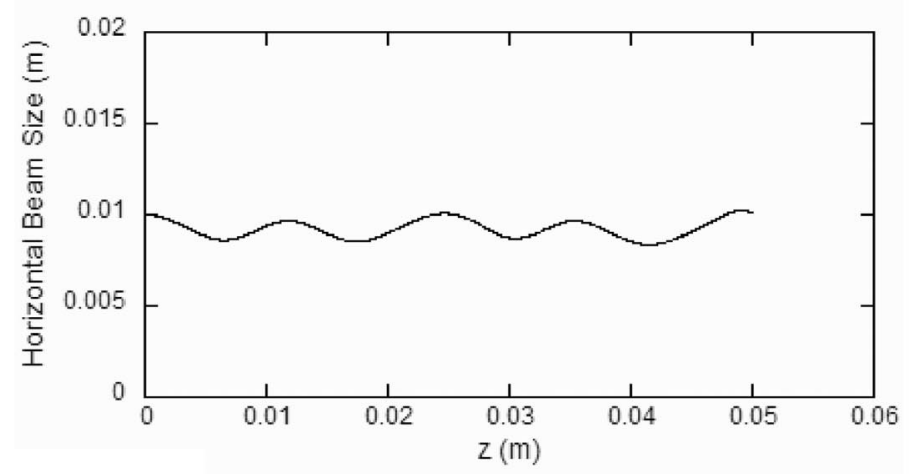

(e)

FIG. 15. Horizontal beam size for PPM focusing augmented by periodic quadrupole (a) 4-mm period, (b) 6-mm period, (c) 8-mm period, (d) 10-mm period, and (e) 12-mm period.

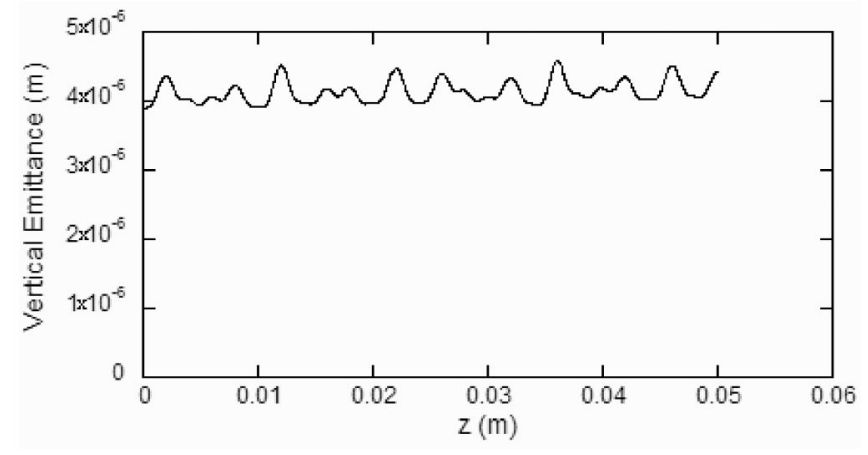

FIG. 16. Vertical emittance, 8-mm period.

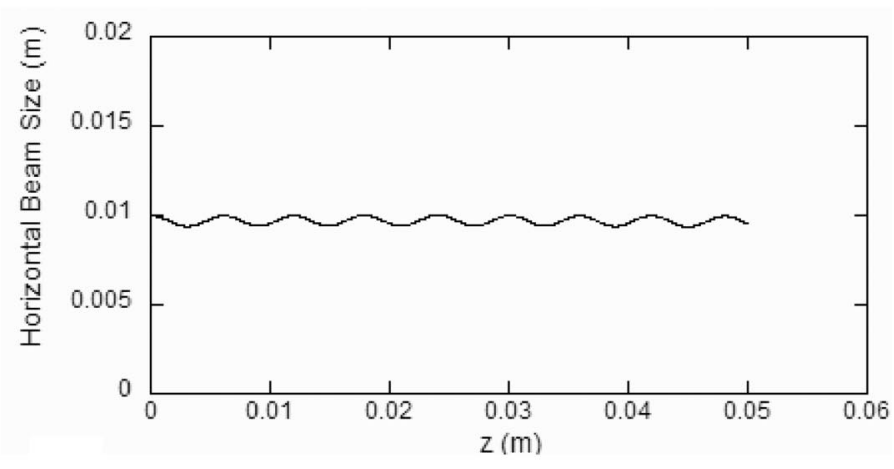

(b)

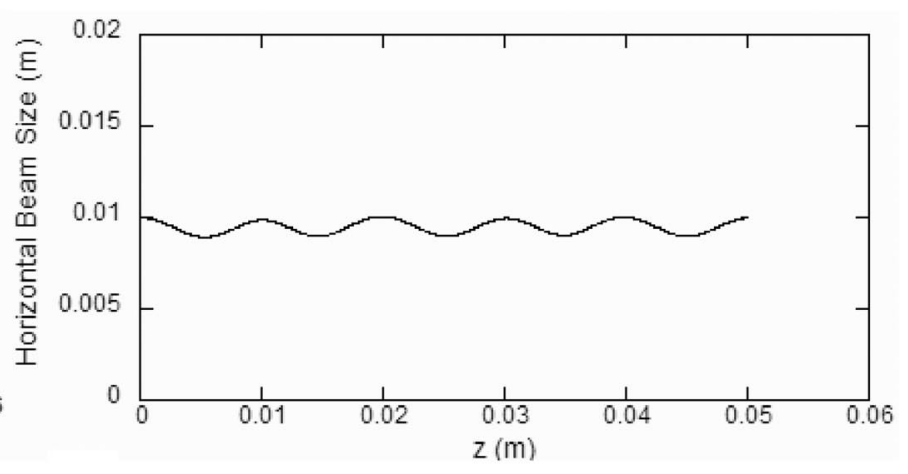

(d) 
[1] B. E. Carlsten, S. J. Russell, L. M. Earley, F. Krawczyk, J. M. Potter, P. Ferguson, and S. Humprhies, Jr., IEEE Trans. Plasma Sci. 33, 85 (2005).

[2] B. E. Carlsten, Phys. Plasmas 8, 4585 (2001).

[3] B. E. Carlsten, Phys. Plasmas 9, 5088 (2002).

[4] B.E. Carlsten, L. M. Earley, F. Krawczyk, S.J. Russell, J.M. Potter, P. Ferguson, and S. Humprhies, Jr., this issue, Phys. Rev. ST Accel. Beams 8, 062001 (2005).

[5] J. T. Mendel, C. F. Quate, and W. H. Yocum, Proc. IRE 42, 800 (1954).

[6] M. A. Basten and J.H. Booske, J. Appl. Phys. 85, 6313 (1999).

[7] J.H. Booske, M.A. Basten, A. H. Kumbasar, T. M. Antonsen, S. W. Bidwell, Y. Carmel, W. W. Destler, V.L.
Granatstein, and D. J. Radack, Phys. Plasmas 1, 1714 (1994).

[8] E. T. Scharlemann, J. Appl. Phys. 58, 2154 (1985).

[9] J.H. Booske, B.D. McVey, and T.M. Antonsen, Jr., J. Appl. Phys. 73, 4140 (1993).

[10] H. P. Freund, H. Bluem, and R. H. Jackson, Nucl. Instrum. Methods Phys. Res., Sect. A 285, 169 (1989).

[11] S. Humphries, S. Russell, B. Carlsten, and L. Earley, IEEE Trans. Plasma Sci. 33, 882 (2005).

[12] B. E. Carlsten, L. M. Young, M. E. Jones, L. E. Thode, A.H. Lumpkin, D.W. Feldman, R. B. Feldman, B. Blind, M.J. Browman, and P.G. O'Shea, IEEE J. Quantum Electron. 27, 2580 (1991). 